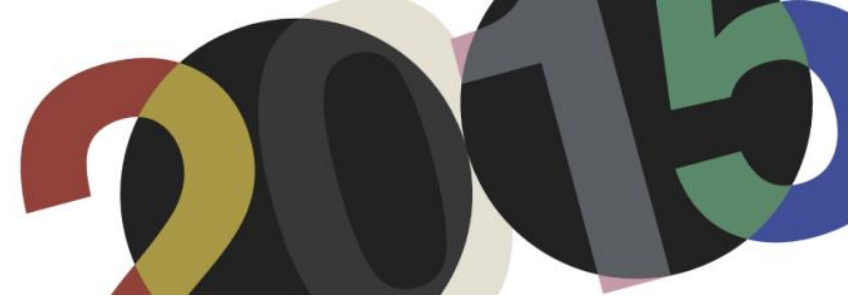

DOI: http://dx.doi.org/10.4995/LC2015.2015.660

\title{
Learning from Le Corbusier
}

\author{
L.J. Duport
}

Ecole Nationale Supérieure d'Architecture de Montpellier

Résumé: Interrogé sur l'enseignement de l'architecture, et bien qu'il ait exposé ses idées sur le sujet principalement dans deux de ses livres : "Précisions» (1930) et «Sur les quatre routes» (1941) Le Corbusier répond : "Je n'ai jamais reçu d'enseignement proprement dit. Je suis autodidacte même dans le sport. ». La formidable puissance didactique de Le Corbusier réside en cinq points : sa formation, son positionnement théorique, son invention de typologies, sa diffusion de l'architecture, sa production prolifique. Ainsi, bien au-delà de l'Euvre Complète Le Corbusier offre à qui veut s'en servir un champ pédagogique particulièrement riche à découvrir et à partager. Aucun programme ne lui a échappé que ce soit les villas ou maisons, le logement collectif, les bureaux, les équipements (publics ou privés), les bâtiments institutionnels, les musées, les usines : tout est matière à invention. Cette invention s'accompagne de sa diffusion de l'architecture, de ses idées, à travers publications et conférences à travers le monde. Mais cela n'est rien comparé à sa production aux échelles variées de l'habitat minimum jusqu'à l'édifice monumental. C'est pourquoi avec le regard porté sur le projet des Quartiers Modernes Frugès construits à Pessac en 1926 nous examinerons comment ce "laboratoire » constitue une expérience pédagogique qui a valeur d'exemplarité et toujours d'actualité.

Abstract: Asked about the architectural education and although he outlined his ideas on the subject mainly in two of his books: "Précisions" (1930) and "Sur les quatre routes" (1941), Le Corbusier replied: "I have never received proper education. I am self-taught even in sport. ". The amazing power of Le Corbusier's didactic resides in five points: his training, his theoretical positioning, his invention of typologies, his diffusion of architecture, his prolific production. Thus, beyond his "Oeuvres Complètes" Le Corbusier offers to whom wants to use it, a rich educational field to discover and share. No program has eluded him whether it is villas or houses, collective housing, offices, facilities (public or private), institutional buildings, museums, factories : everything is material for invention. This invention is accompanied with his diffusion of architecture, of his ideas in books or lectures all over the world. But this is nothing compare to his production to various scales from the minimum housing to the monumental building. Therefore with the close look on the Modern Quarters Frugès project built in Pessac in 1926 we will examine how this "laboratory" is an educational experience that has of exemplarity value and is still relevant today.

Mots-clés: Enseignement, Habitat, Patrimoine XXe, polychromie, Restauration. Keywords: Education, Housing, Heritage XXe, Polychromie, Restoration. 
Lorsqu'on l'interroge sur l'enseignement de l'architecture, et bien qu'il ait exposé ses idées sur le sujet principalement dans deux de ses livres : «Précisions » (1930) et « Sur les quatre routes » (1941), Le Corbusier répond : «Je n'ai jamais reçu d'enseignement proprement dit. Je suis autodidacte même dans le sport ${ }^{1}{ } »$

La formidable puissance didactique de Le Corbusier réside en cinq points : sa formation, son positionnement théorique, sa diffusion de l'architecture, sa production prolifique, son invention de typologies. Ainsi bien au-delà de l'CEuvre Complète, Le Corbusier offre à qui veut s'en servir un champ pédagogique particulièrement riche à découvrir et à partager.

Sa formation tout d'abord où l'on se rend compte que tout est exploité à dessein : que ce soit par son apprentissage même, par ses croquis et récits de voyages, par ses rencontres ou encore à l'occasion de la lecture de ses premières publications. L'influence de Charles l'Eplattenier (1874-1946) qui dirige l'Ecole d'Art de la Chaux de Fonds sera décisive. C'est lui qui suggère à Charles Edouard Jeanneret de voyager et surtout qui obtiendra la confiance d'un premier client Louis Fallet, pour qui le jeune Jeanneret construira sa première villa à la Chaux-de-Fond (avec l'architecte Chapellaz en 1906).

Cette formation restera fondamentale dans la capacité de Le Corbusier à choisir sa vocation, s'écartant de l'avenir tout tracé qui lui était réservé de graveur de montres. Ses voyages en Italie (1907), en Allemagne (1910) ou encore en Orient (1911) font du jeune Jeanneret un infatigable dessinateur, photographe mais le propulse aussi chez les meilleurs architectes du moment, Joseph Hoffman, Auguste Perret et Peter Behrens. Le Corbusier va ainsi «parcourir le monde à la recherche d'une identité et d'une culture d'architecte ${ }^{2} »$.

Pour lui «Quand on voyage et qu'on est praticien des choses visuelles : architecture, peinture ou sculpture, on regarde avec des yeux et on dessine afin de pousser à l'intérieur, dans sa propre histoire, les choses vues. Une fois les choses entrées par le travail du crayon, elles restent pour la vie; elles sont écrites, elles sont inscrites (...). Dessiner soi-même, suivre des profils, occuper des surfaces, reconnaitre des volumes, etc. (...) c'est d'abord regarder, c'est être apte peut-être à observer, apte peut-être à découvrir...A ce moment-là le phénomène inventif peut survenir. On invente et même on crée; tout l'être est entrainé dans l'action; cette action c'est le point capital. $»^{3}$

Ses voyages de jeunesse augmentés par sa capacité d'interprétation autorise un positionnement théorique qu'il ne va cesser de publier que ce soit depuis «Vers une architecture » (1923) jusqu'à « Mise au point» (1966) en passant par «Manière de penser l'urbanisme» (1946) ou «Le modulor» (1950). Mais c'est certainement son implication dans "la revue L'Esprit Nouveau qu'il créa en 1920 avec Amédée Ozenfant ${ }^{4}$ et dirigea avec lui et Paul Dermée ${ }^{5}$ jusqu'en 1925, date à laquelle la revue cessa de paraître. Il y écrivit douze articles qu'il rassembla dans un livre, Vers une architecture, ${ }^{6}$ publié en 1923. (...) Bien que ce livre se ressente, dans son style, de la juxtaposition d'articles écrits pour une revue militante il n'en demeure pas moins que Le Corbusier y exposa alors l'essentiel de ses théories, et ne fit, par la suite, que les répéter, les préciser, et les développer dans ses autres écrits ${ }^{7}$

\footnotetext{
${ }^{1}$ Entretien avec les étudiants des écoles d'architecture, Le Corbusier, Denoël, Paris, 1943

${ }^{2}$ Duboy Philippe, Le Corbusier, Croquis de voyages et études, textes choisis, Louis Vuitton, Paris 2009, p.15

${ }^{3}$ Le Corbusier, L'atelier de la recherche patiente, Vincent et Fréal, Paris, 1960, p.37, cité par P.Duboy

${ }^{4}$ Amédée Ozenfant (1886-1966) peintre français, co-fondateur (avec Le Corbusier) de la revue L’Esprit Nouveau et du purisme (en réaction au cubisme)

${ }^{5}$ Paul Dermée (1886-1951), écrivain, poète et critique littéraire, directeur de la revue L'Esprit Nouveau

${ }^{6}$ Le Corbusier, Vers une architecture, Éditions Crès, Collection de "L'Esprit Nouveau", Paris, 1923

${ }^{7}$ Guiton Jacques Le Corbusier, Textes choisis Architecture et urbanisme, Le Moniteur, Paris 1982
} 
Cette invention s'accompagne de sa diffusion de l'architecture et de ses idées à travers des publications comme l'Esprit Nouveau (1920) mais surtout grâce à des conférences à travers le monde, à la fois au sein des CIAM depuis l'origine (1928) ou avec des voyages et des conférences données aux USA, en Russie et en Amérique Latine, en particulier à Buenos Aires en 1929.

Cela n'est rien en comparaison avec sa production prolifique que ce soit de projets non construit à ses nombreuses réalisations aux échelles variées dans le monde entier, de l'habitat minimum, avec le Cabanon à Roquebrune Cap Martin (1952) jusqu'à l'édifice monumental comme le palais de l'Assemblée à Chandigarh (1955). Au-delà de sa propre production elle-même, Le Corbusier était un inventeur de typologies. Aucun programme ne lui a échappé, que ce soit les villas ou maisons, le logement collectif, les bureaux, les équipements (publics ou privés), les bâtiments institutionnels, les musées, les usines. Tout ou presque est matière à invention.

Avec le projet des Quartiers Modernes Frugès construits à Pessac en 1928 nous examinerons pourquoi ce « laboratoire » constitue une expérience pédagogique qui a valeur d'exemplarité et toujours d'actualité.

\section{Les Quartiers Modernes Frugès}

La construction de cette cité entre 1924 et 1926 est le fruit de la rencontre de deux personnalités : un industriel sucrier, Henry Frugès, et un architecte, Le Corbusier. Henry Frugès, curieux de toutes les innovations artistiques et architecturales du moment, souhaitait loger des ouvriers sur "une vaste prairie entourée de bois de pins, en y édifiant une cité-jardin". Le Corbusier, porté par un esprit d'avant-garde, avait déjà abordé les problèmes liés à l'urbanisme. l'habitat et les maisons standardisées.
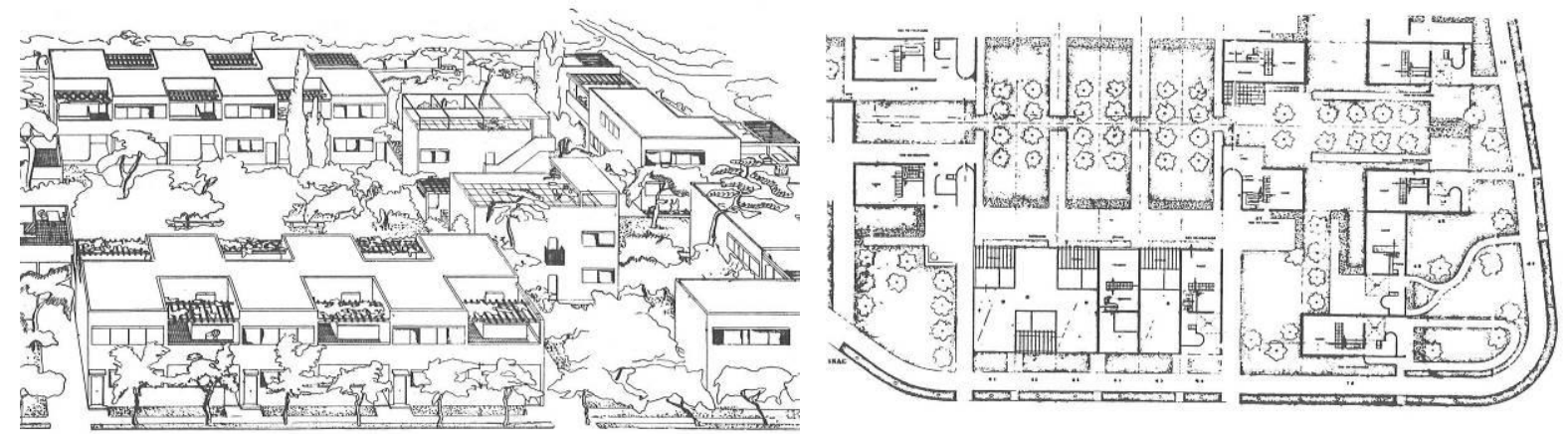

1. - 2. Vers une architecture p.212 et 213 OFLC-ADAGP

La Cité Frugès est née de leurs échanges, et particulièrement de la réflexion de l'architecte, qui adopta des principes strictement fonctionnels et des formes géométriques simples. Avant-gardiste formellement et par sa dimension sociale, la cité est à l'époque une véritable révolution, tant sur le plan de l'habitat social que sur celui de l'architecture C'est en termes de confort et d'esthétique, pour des logements modestes construits à l'époque, que l'architecte se positionna en véritable précurseur. Les modèles d'habitations conçus ici illustrent les cinq points de l'architecture moderne : les pilotis, le toit-terrasse, le plan libre, la fenêtre en longueur, la façade libre.

L'ambition d'origine se trouve diminuée : sur les 150 logements initialement prévus, seulement 51 seront réalisés en 1926 et resteront vides pour leur grande majorité jusqu'en 1929.

De même sa réception se révèle un échec, en raison de l'accueil mitigé que cette expérimentation tournée vers la modernité suscita. Bousculant les esprits, la cité Le Corbusier de Pessac fut accompagnée de nombreuses critiques. 
Parmi les nombreux documents aujourd'hui disponibles et hormis les originaux produits par l'Atelier Le Corbusier trois publications essentielles rendent compte du caractère innovant de la réalisation de la Cité Frugès et de sa fortune critiques.
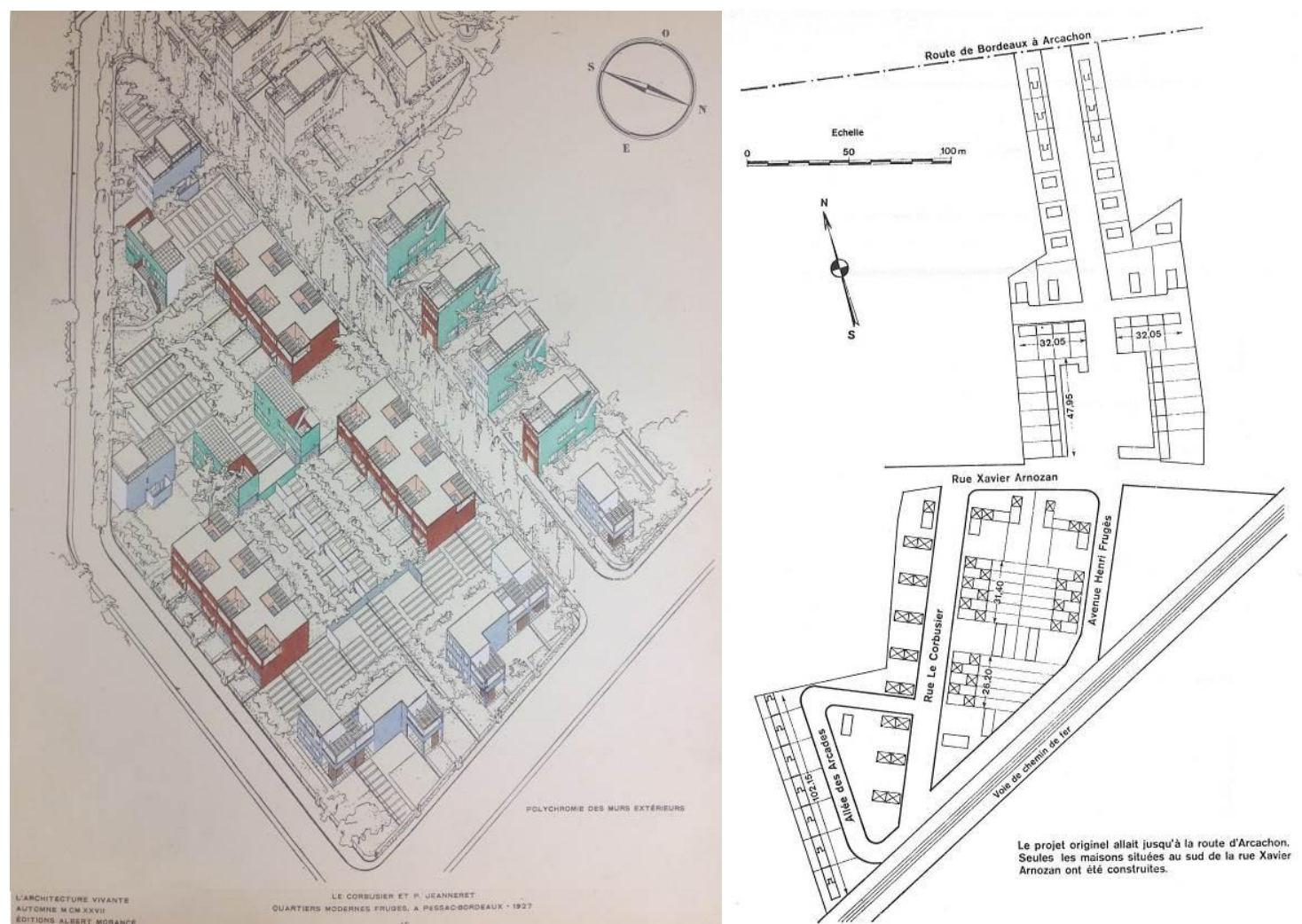

3. Quartiers Modernes Frugès in l'Architecture Vivante CFLC-ADAGP

4. P.Boudon Pessac de Le Corbusier p.38 CFLC-ADAGP

La première est le catalogue de l'exposition Le Corbusier at Pessac ${ }^{8}$ à Harvard sous la direction d'Eduard Sekler et de Brian Brace Taylor qui explique la genèse des QMF, la deuxième est le livre de Pessac de Le Corbusier par Philippe Boudon ${ }^{9}$, la troisième est le guide intitulé Le Corbusier : Les Quartiers Modernes Frugès par Marylène Ferrand, Bernard Le Roy et Jean Luc Veyret ${ }^{10}$

Ainsi donc dans Le Corbusier at Pessac Eduard Sekler et Brian Brace Taylor expliquent la genèse du projet d'un point de vue à la fois historique et théorique depuis les analyses effectuées par le jeune Jeanneret à la Bibliothèque Nationale en 1914-1915 de l'« Enquête sur les conditions de l'Habitation en France, Les Maisons Types» par Alfred de Foville de $1894{ }^{11}$ en passant par l'élaboration des plans types de la maison DOM-INO jusqu'à l'intérêt porté aux études de Tony Garnier pour sa Cité Industrielle comme le montre la correspondance entre Jeanneret et Garnier de 1915.

\footnotetext{
${ }^{8}$ Sekler Eduard \& Taylor Brian Brace Le Corbusier at Pessac, The Search for Systems and Standards in the Design of Low Cost Housing, Catalogue de l'exposition éponyme 12 0ctobre 30 Novembre 1972, Carpenter Center for the Visual Arts, Harvard University, en collaboration avec la Fondation Le Corbusier.

${ }^{9}$ Boudon Philippe, Pessac de Le Corbusier, première édition, Bordas, Paris 1969 suivi d'une édition augmentée, Dunod, Paris 1985

${ }^{10}$ Ferrand Marylène, Feugas Jean Pierre, Le Roy Bernard, Veyret Jean Louis, Le Corbusier : Les Quartiers Modernes Frugès, Fondation Le Corbusier, Paris, Birkhäuser, Bâle, Boston, Berlin

${ }^{11}$ De Foville Alfred, Enquête sur les conditions de l'Habitation en France, Les Maisons Types, Paris 1894
} 
Mais l'implication de l'architecte va bien au-delà de la théorie car le jeune Jeanneret va être également architecte consultant pour la S.A.B.A (Société d'Application du Béton Armé) l'entrainant ainsi sur des programmes divers comme le château d'eau de Podensac, un arsenal à Toulouse, une usine hydro électrique ou encore une centrale électrique à Saintes. Ce dernier programme oblige Jeanneret à « résumer ses recherches sur les habitations pour les ouvriers. Sa solution de logements proches de la centrale électrique d'Avril 1917 est la première d'une série de projets de logements ouvriers que Jeanneret entreprit dans la France rurale. Un aspect de ce programme particulier était que les ouvriers et contremaîtres de nuit devaient vivre avec leur famille sur le site à proximité de la machine en fonctionnement constant. Les questions sociales ainsi que la nature pratique soulevée par cette situation dans laquelle la production de la machine et le travail, la famille, la vie et les loisirs de l'hommes sont intimement liés, constituent finalement un thème central des théories de Le Corbusier sur l'architecture et

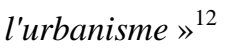

A cela s'ajoute l'idéologie de la Cité Jardin inspirée du modèle anglais des architectes anglais Barry Parker et Raymond Uwin que Jeanneret expérimente dans un projet à la Chaux de Fond et surtout en juin 1917 à Saint Nicolas d'Aliermont avec un projet pour la construction de 25 logements ouvriers. Pour répondre au souhait du commanditaire, l'architecte propose 3 types de maisons avec un traitement différent de la façade afin de différencier les maisons des contremaîtres de celles des ouvriers. Composées de deux niveaux, les habitations sont percées de nombreuses fenêtres pour laisser passer la lumière. Les maisons sont entourées d'un jardin et d'une courette et disposées de part et d'autre d'une voie unique conduisant de la coopérative à une placette en cul-de-sac. La répartition est régulière et aérée, d'une faible densité, laissant une surface large à la verdure.

Une première expérience est menée avec Henry Frugès à Lège constituant l'antichambre des Quartiers Modernes Frugès. Construit en 1923/1924 à Lège-Cap-Ferret le lotissement de six petites maisons, est réalisé pour le compte de l'industriel sucrier afin d'y loger les ouvriers employés à la scierie locale. Le Corbusier propose des maisons minimum selon un type $\mathrm{A}$ et un type $\mathrm{B}$, basées sur des dimensions et une géométrie qui caractérisent « une évolution de ses projets précédents à Saintes et Saint Nicolas d'Aliermont ${ }^{13 .}$

Pour les Quartiers Modernes Frugès à Pessac les premiers plans conçus dès 1924 développent un changement d'échelle ainsi que d'autres expérimentations particulièrement pertinentes comme la polychromie et les assemblages de typologies variées selon les différents secteurs.

«Je vous autorise à réaliser dans la pratique vos théories, jusque dans leurs conséquences les plus extrêmes, je désire atteindre à des résultats vraiment concluants dans la réforme de l'habitation à bon marché : Pessac doit être un laboratoire ${ }^{14} \gg$. Henry Frugès passe commande à l'architecte Le Corbusier en 1924 souhaitant implanter une " cité-jardin " pour loger ses ouvriers.

Ainsi Le Corbusier, dont les préoccupations architecturales du moment sont le logement de masse, va travailler avec Pierre Jeanneret sur le terrain de Pessac Le premier plan comprend 135 maisons, réparties en quatre secteurs. La composition s'inspire des plans des cités jardins anglaises : hiérarchie, diversité et qualité des espaces urbains, les maisons sont composées comme des objets isolés aux formes pures. Les deux architectes définissent un modèle standard, les éléments sont tous identiques sur chaque cellule : ossature, fenêtres (en longueur), escaliers assemblés perpendiculairement à l'intérieur, parallèles à la plus grande façade à l'extérieur. Les maisons se combineront entre elles selon leur type. Mais avant tout, le chantier est un terrain d'application de la méthode d'organisation du travail industriel, standardisé dans ses éléments : la même « cellule » de $5 \mathrm{~m}$ par 5

\footnotetext{
${ }^{12}$ Le Corbusier at Pessac, The Search for Systems and Standards in the Design of Low Cost Housing, p.13

${ }^{13}$ Benton Tim, «Pessac and Lège revisited : standards, dimensions and failures » in Massilia, 2004 p.68

${ }^{14}$ Le Corbusier \& Pierre Jeanneret, Euvres Complètes 1910-1929 volume I p.78
} 
mètres et de 2,50m par 5 mètres, la même fenêtre, le même escalier, le même système de chauffage, les mêmes équipements de cuisine et de salles de bains.

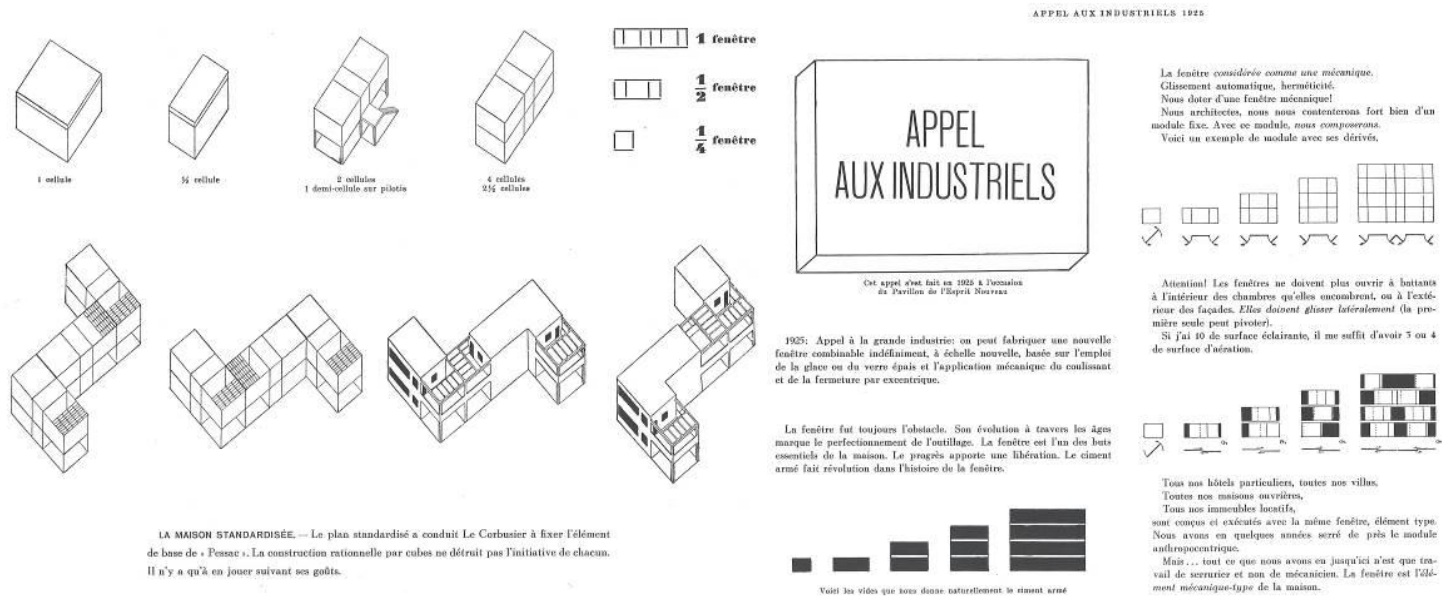

5. Le Corbusier Euvres Complètes 19101929 p.69@FLC-ADAGP

6. Le Corbusier CEuvres Complètes 19101929 p.77@FLC-ADAGP

\section{La polychromie}

«Pessac devient une promenade urbanistique bien ordonnée, une sorte de pendant à la promenade architecturale des Villas la Roche Jeanneret dont la polychromie intérieure a été déterminée sensiblement au même moment ${ }^{15}$

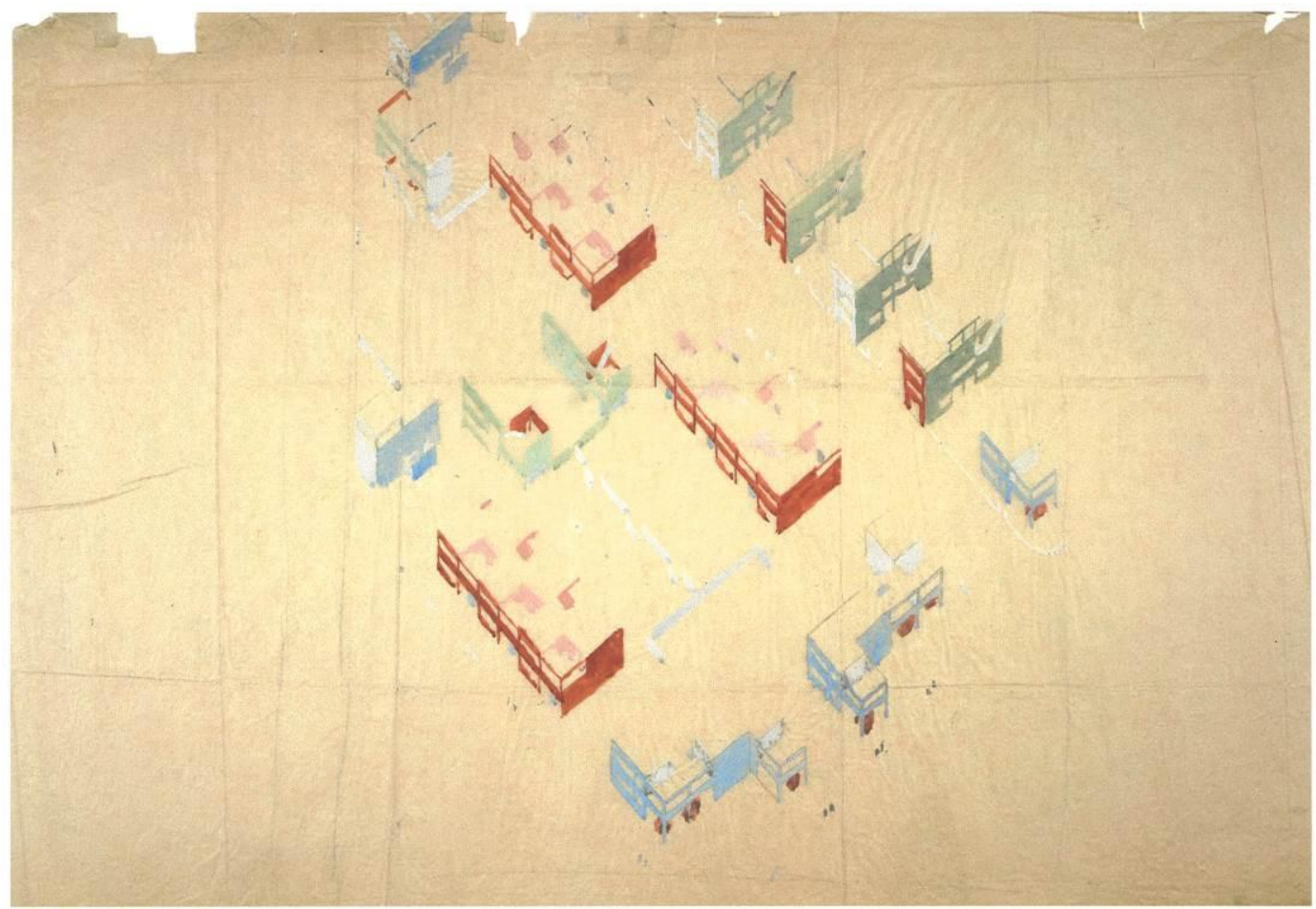

7. Le Corbusier et Pierre Jeanneret, Quartiers Modernes Frugès, Pessac, étude de polychromie, 1924. Gouache sur calque, 78,2 X $110 \mathrm{~cm}$. Fondation Le Corbusier CFLC-ADAGP

\footnotetext{
${ }^{15}$ Le Corbusier at Pessac, The Search for Systems and Standards in the Design of Low Cost Housing, p.14
} 

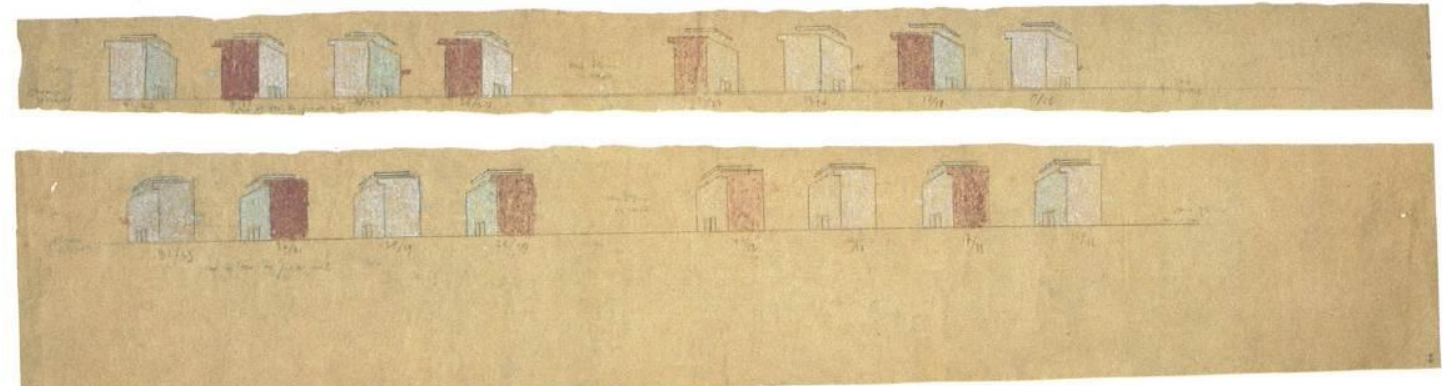

8. Le Corbusier et Pierre Jeanneret, Quartiers Modernes Frugès, Pessac, étude de polychromie, 1924. Gouache sur calque, $41,8 \mathrm{X} 114,2 \mathrm{~cm}$. Fondation Le Corbusier @FLC-ADAGP

C'est ainsi avec les QMF, la première fois que Le Corbusier utilise la polychromie extérieure, justifiant que « la couleur pouvait nous apporter l'espace. Considérer la couleur comme apporteuse d'espace. Voici comment nous avons établi des points fixes : certaines façades peintes en terre de Sienne brûlée pur.

«Nous avons fait fuir au loin des lignées de maisons : bleu outremer clair. Nous avons confondu certains secteurs avec le feuillage des jardins et de la forêt : façades vert pâle ${ }^{16 .}$

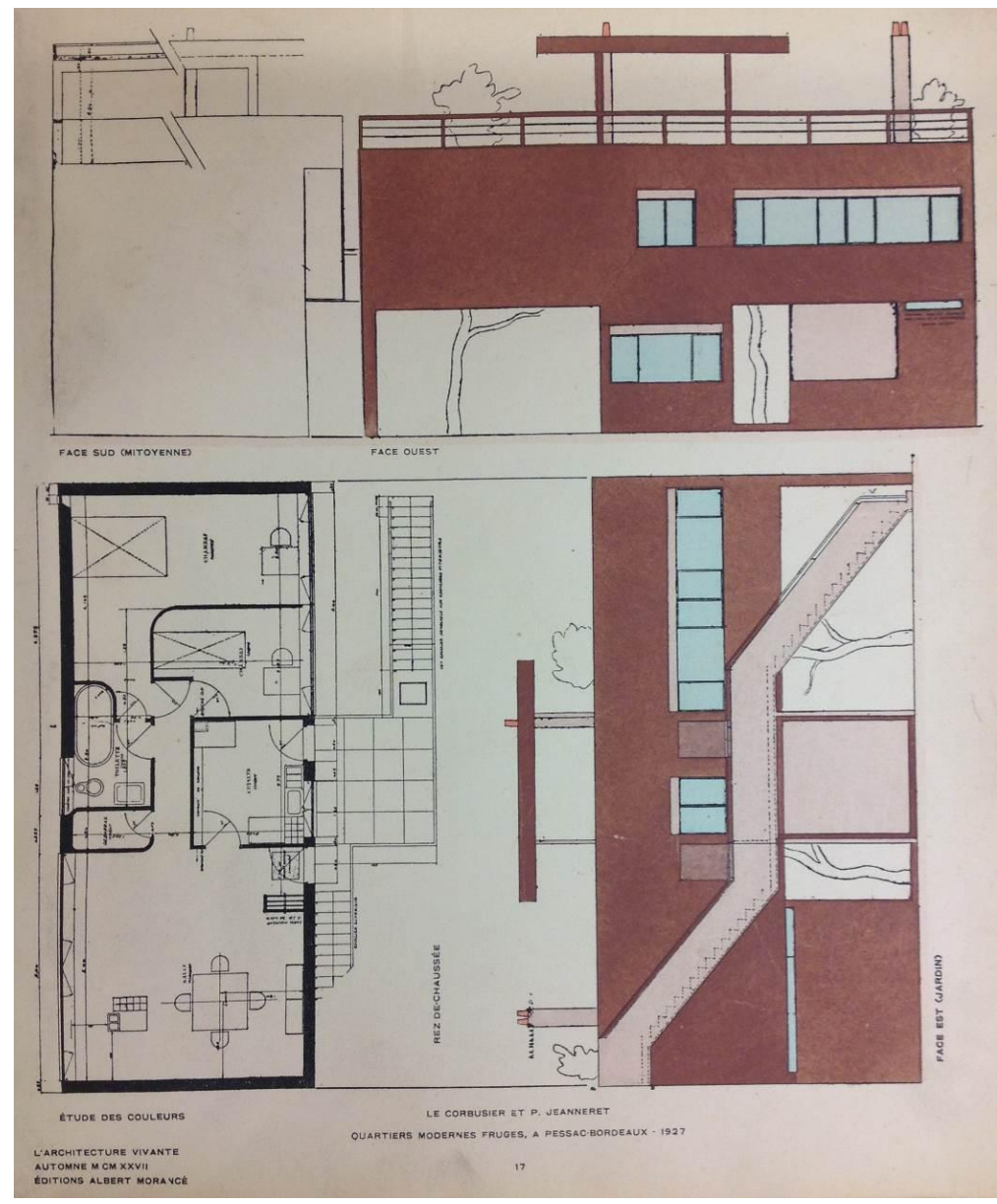

9. Quartiers Modernes Frugès Etude des couleurs in l'Architecture Vivante, 1927 OFLC-ADAGP

\footnotetext{
${ }^{16}$ Le Corbusier \& Pierre Jeanneret, Euvres Complètes 1910-1929 volume I p.86
} 
Enfin et surtout «il se dégage des constructions de Pessac une esthétique inattendue, neuve. Mais cette esthétique est licite, conditionnée par les impératifs de la construction d'une part, et d'autre part, par les bases primordiales de la sensation architecturale, le volume. Les prismes qui se dressent les uns à côté des autres obéissent à des règles de mise en proportion, rapports que nous avons cherché à rendre éloquents et harmonieux. Nous avons aussi appliqué une conception entièrement neuve dans la polychromie, poursuivant un but nettement architectural: modeler l'espace grâce à la physique même de la couleur, affirmer certaines masses du lotissement, en faire fuir certaines autres, en un mot composer avec la couleur comme nous avions fait avec les formes. C'était ainsi conduire l'architecture dans l'urbanisme ${ }^{17}$.
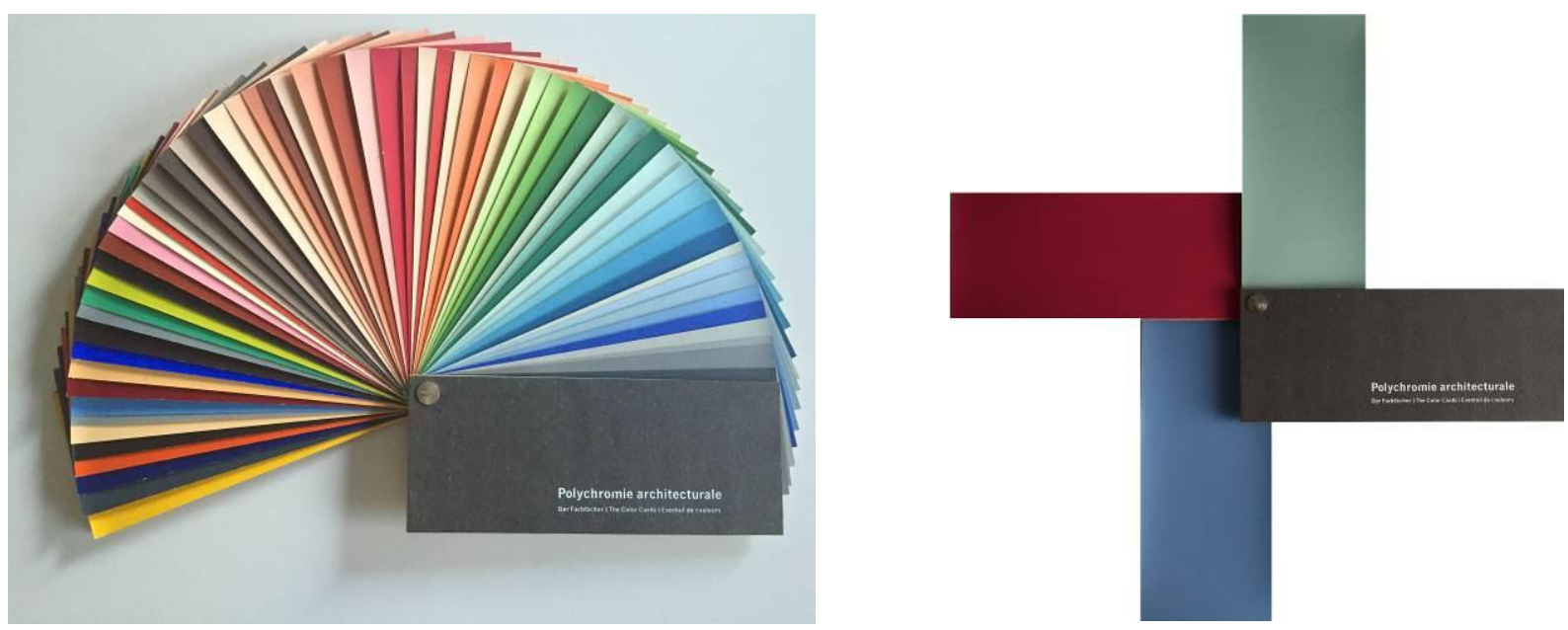

10. Claviers de couleurs Salubra de Le Corbusier @FLC-ADAGP

11. Couleurs des Quartiers Modernes Frugès @FLC-ADAGP

Il faut voir ici les fondements de la polychromie architecturale de Le Corbusier qu'il développera avec le nuancier Salubra en deux étapes, l'une de 43 coloris baptisé «claviers de couleurs » en 1931 puis avec une seconde gamme de 20 couleurs en 1959, cette dernière étant employée dans les projets comme les Maisons Jaoul, ou d'autres projets issus du « brutalisme».
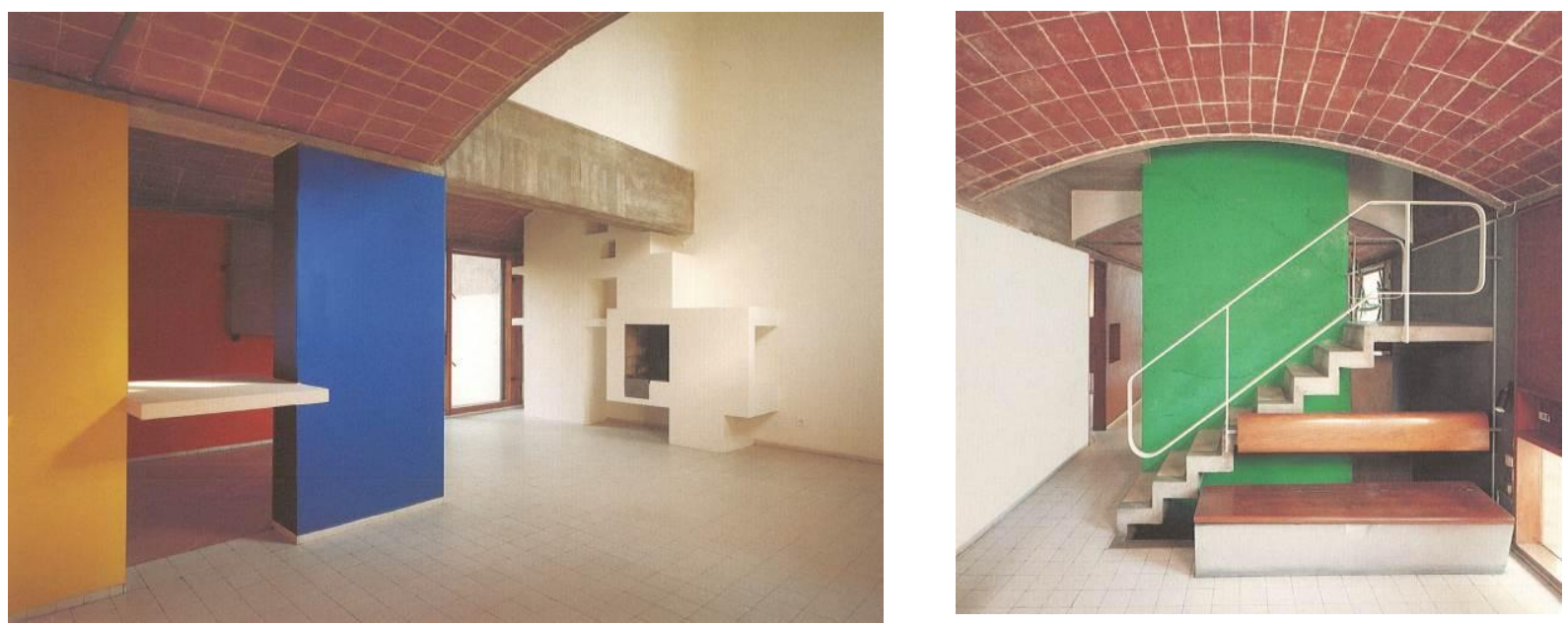

12. Maisons Jaoul, Le Corbusier, Maison A, salon @FLC-ADAGP

13. Maisons Jaoul, Le Corbusier, Maison A, entrée OFLC-ADAGP

\footnotetext{
${ }^{17}$ Le Corbusier, exposé lors de l'inauguration des Quartiers Modernes Frugès, à l'occasion de la visite officielle de M. de Monzie, ministre des Travaux Publics à Pessac le 13 juin 1926, cité par Philippe Boudon, Pessac de Le Corbusier, p.148
} 


\section{Construction}

Le chantier débute en 1925, et permet à Le Corbusier de composer avec les volumes, la lumière et la polychromie: la couleur est choisie en fonction de l'intensité lumineuse et de l'orientation des façades. L'utilisation du canon à ciment projeté sur ossature expérimentée à Lège s'avéra un choix coûteux et erroné. Un mélange extrêmement riche en ciment était exigé, et dans le même temps, il était presque impossible d'arriver à un mur d'épaisseur optimale et uniforme. La construction à Pessac sera finalement réalisée en blocs manufacturés, le ciment projeté servira seulement pour les murs courbes, les chais et murs des jardins. Mais la réalisation de l'opération bute sur de nombreux obstacles : défaillance de l'entreprise locale, difficultés du chantier, techniques de construction inadaptée. Achevées en 1926, les maisons restent vides jusqu'en 1929. Cette occupation tardive, due à l'absence pour des raisons administratives d'adduction d'eau, bloquant la vente des maisons, entrainant la faillite de leur commanditaire. Sur les 135 logements prévus à l'origine, seuls 53 seront finalement réalisés. Cependant les quartiers modernes Frugès sont d'une importance capitale d'un point de vue historique, dans la mesure où ils sont à la fois la première cité de logements sociaux réalisés par le Corbusier et son premier projet d'envergure.

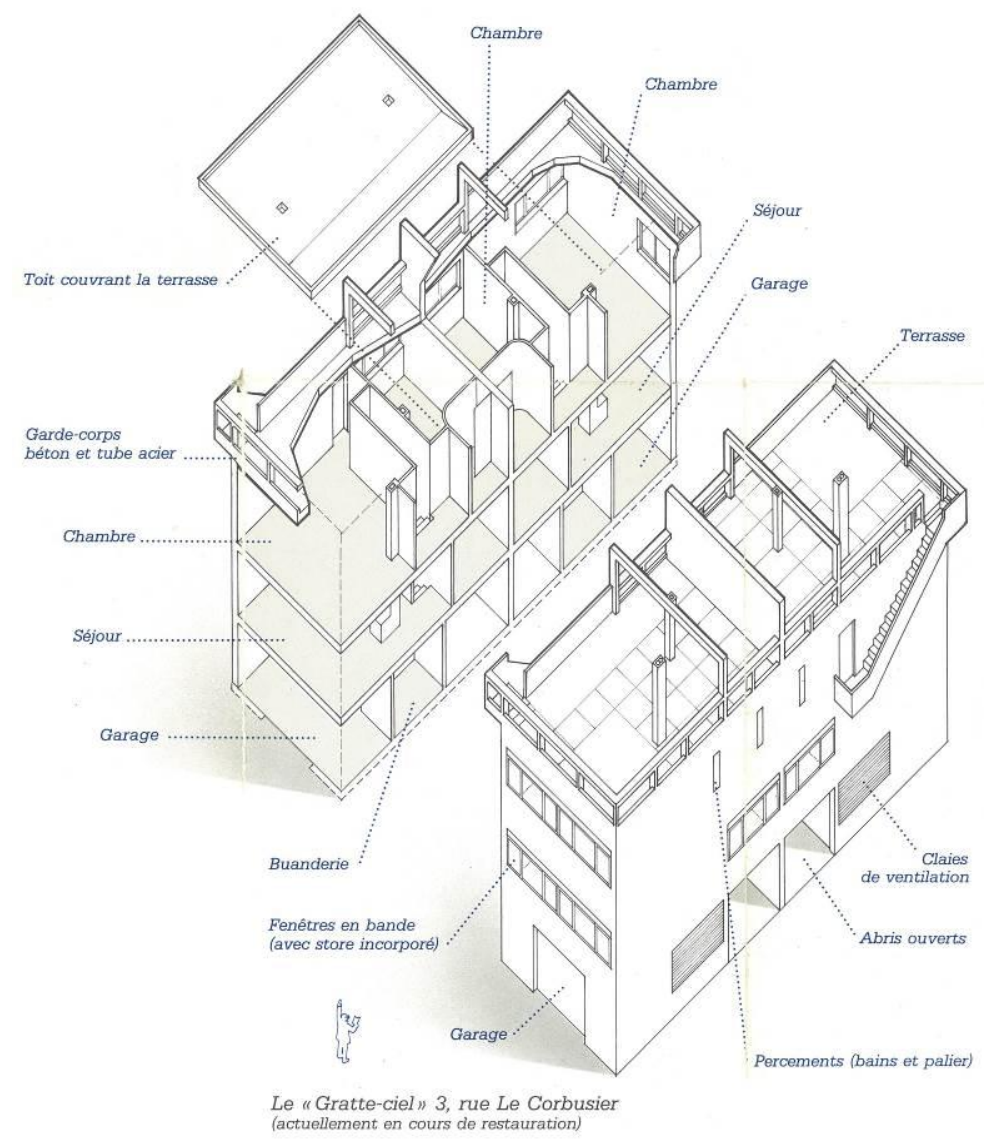

14. Extrait du dépliant présentant l'exposition «L'aventure Le Corbusier », Paris 1987, CFLC-ADAGP

Le parcours dans la cité Frugès permet de découvrir la cinquantaine d'habitations déclinées en 6 typologies différentes : "zigzag", "quinconce", "jumelle", "gratte-ciel", "arcade" et "isolée". 
Gratte-ciel :

Son nom est du à sa hauteur : la plus élevée de toutes les compositions. Cette maison est l'emblème du quartier, les huit bâtiments marquant l'axe principal de l'ensemble de l'opération. Chaque volume comprend deux logements dos à dos sur quatre niveaux, avec une toiture terrasse accessible par un escalier extérieur. Au rez-dechaussée : abri, buanderie et garage, premier étage : entrée, séjour et cuisine, second : deux chambres et sanitaires. Elle possède ainsi la plus grande surface habitable de tous les types de maisons : $110 \mathrm{~m}^{2}$.Côté rue, en alternance les couleurs sont terre de Sienne ou blanche, vert pâle et blanc pour les façades latérales.
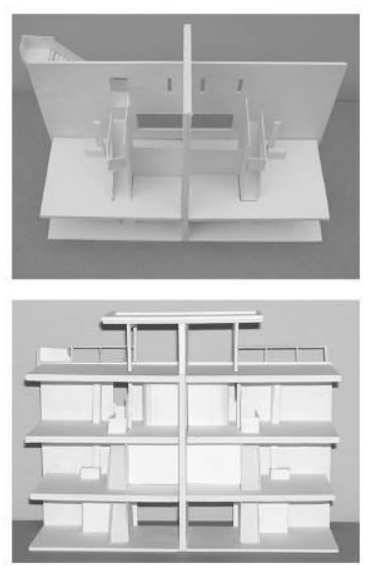
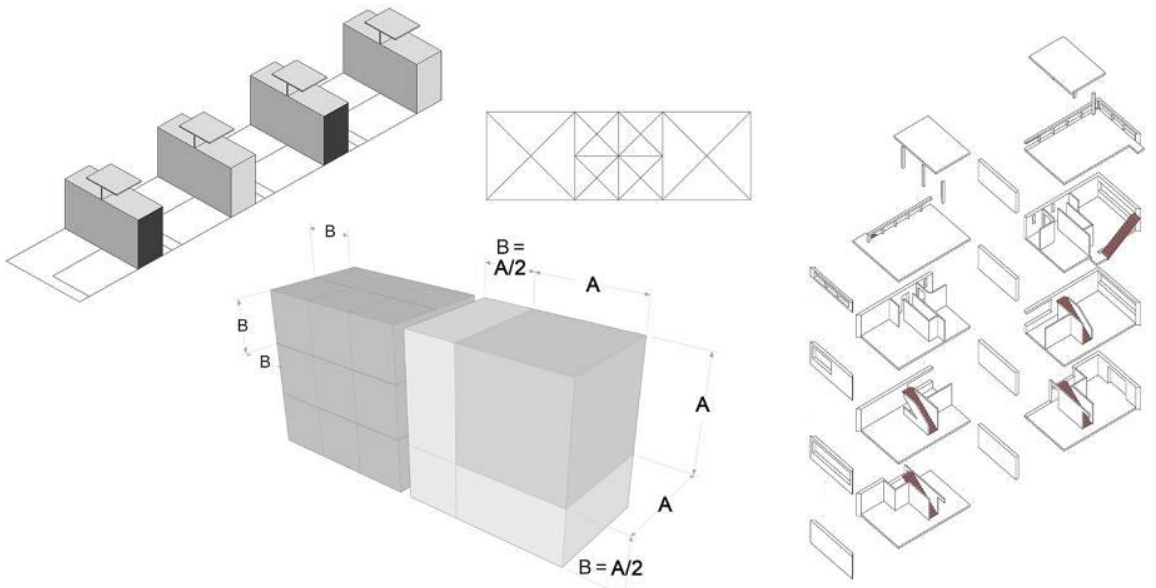

15. Cité Frugès, Les Maisons Gratte Ciel travaux d'étudiants Studio Laurent Duport, ENSAM

Arcades :

L'unité de sept habitations compose la limite ouest de la cité en un long mur percé de grands vides donnant sur le parc voisin La terrasse prend forme au rez-de-chaussée, sous une arcade, comme un patio, dans le prolongement de la maison de $85 \mathrm{~m}^{2}$ de surface habitable. La cuisine, le séjour et l'entrée, le chai, la buanderie sont au rez-dechaussée, les pièces de service sont placées sous voûte. L'étage comporte trois chambres et la salle de bains. La façade côté rue est terre de Sienne, la terrasse ainsi que la voûte sont blanches.
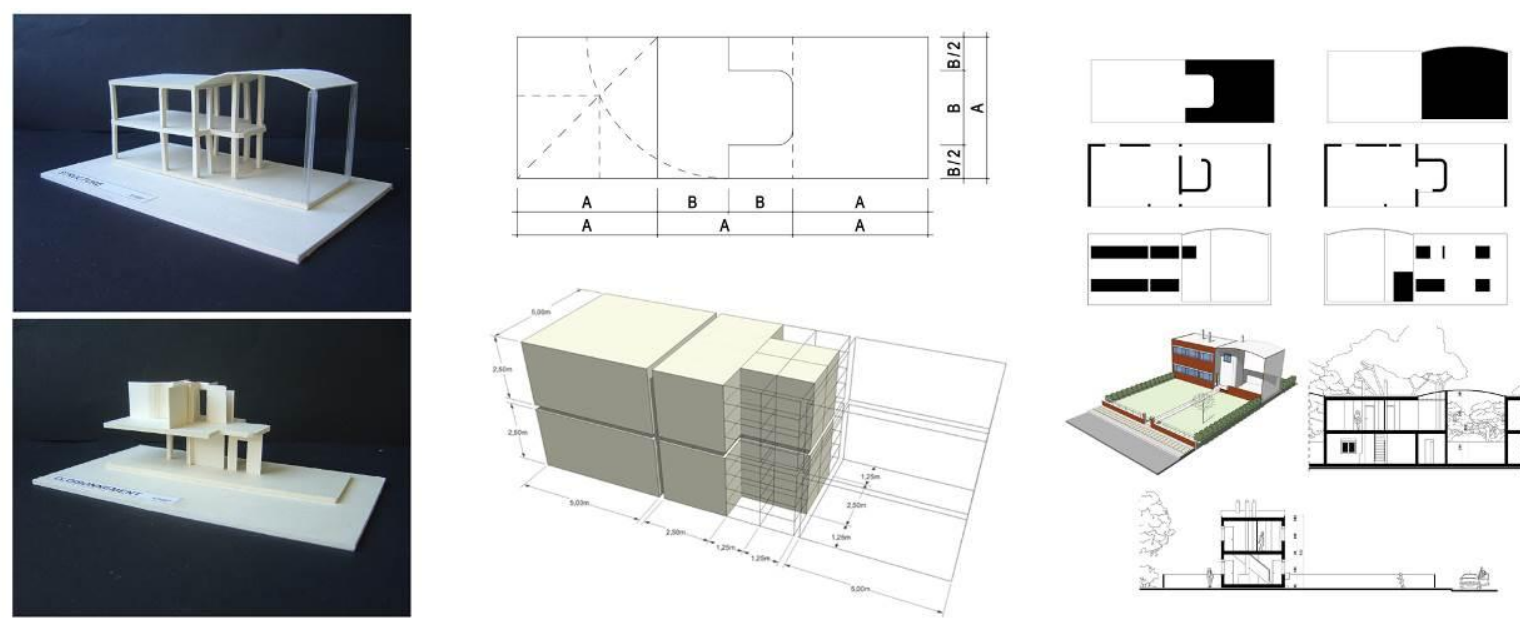

16. Cité Frugès, Les Maisons Arcades travaux d'étudiants Studio Laurent Duport, ENSAM

Quinconce : 
Ce sont des bandes de six logements orientés est-ouest, inversés les uns aux autres ce qui permet une isolation phonique. A l'étage les terrasses en retrait alternent avec la chambre principale. Ici les pièces à vivre se situent au rez-de-chaussée, seule la chambre demeure à l'étage, accompagnée de la salle de bains, et de la terrasse. La surface habitable est de $80 \mathrm{~m}^{2}$. Le chai du rez-de-chaussée est bleu outremer clair, les murs de la terrasse sont couleur terre de Sienne et le reste blanc.
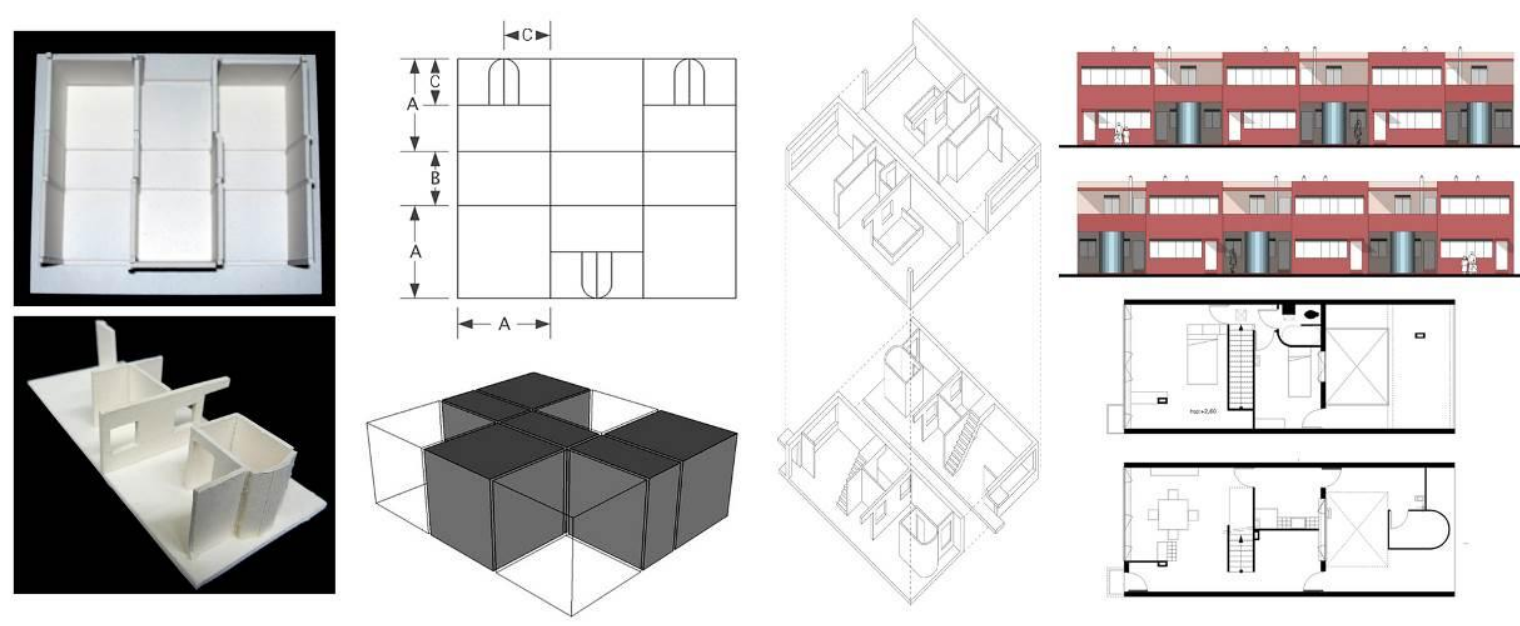

17. Cité Frugès, Les Maisons Quinconces travaux d'étudiants Studio Laurent Duport, ENSAM

Zig Zag :

Semblables au type "Quinconces", deux blocs "zig-zag" sont composés de trois logements accolés mais orientés différemment. Ils sont largement ouverts sur le grand côté de la construction. Les façades sont de couleur bleu outremer clair et blanc. La surface habitable est de $80 \mathrm{~m}^{2}$ répartie sur deux niveaux : au rez-de-chaussée se trouvent le chai, le parloir, la cuisine et la pièce à vivre, et à l'étage deux chambres, les sanitaires et la terrasse.
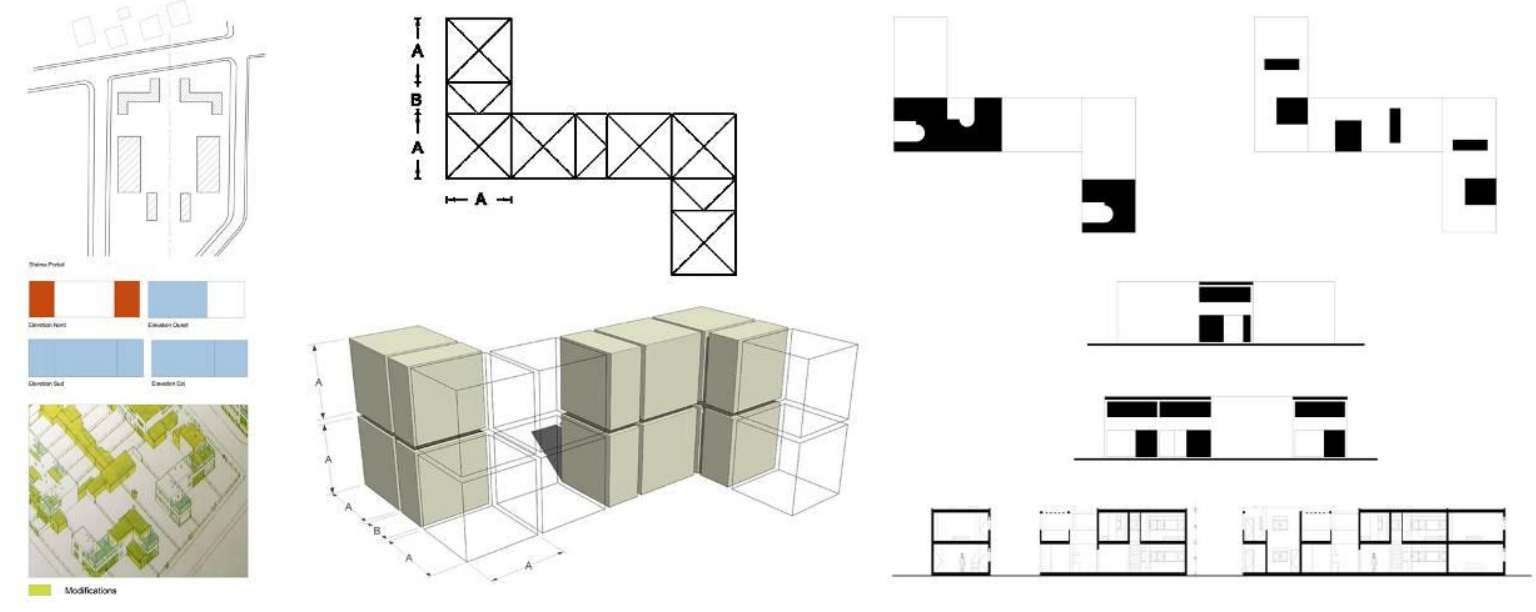

18. Cité Frugès, Les Maisons Zig Zag travaux d'étudiants Studio Laurent Duport, ENSAM

Vrinat : 
Seules deux habitations selon cette typologie étaient prévues dans la cité, une seule a été construite et a bénéficié d'une restauration en 1993. Ce modèle s'organise sur deux niveaux, avec la toiture-terrasse accessible par un escalier extérieur latéral. Le système constructif est celui qui se rapproche le plus des cinq points de Le Corbusier. La surface habitable est de $70 \mathrm{~m}^{2}$. Les pièces habitables sont au 1er étage, au 2ème se situe la terrasse. Les façades sont vert clair et blanches, l'escalier blanc sur sa face extérieure et terre de sienne brulée sur sa face intérieure
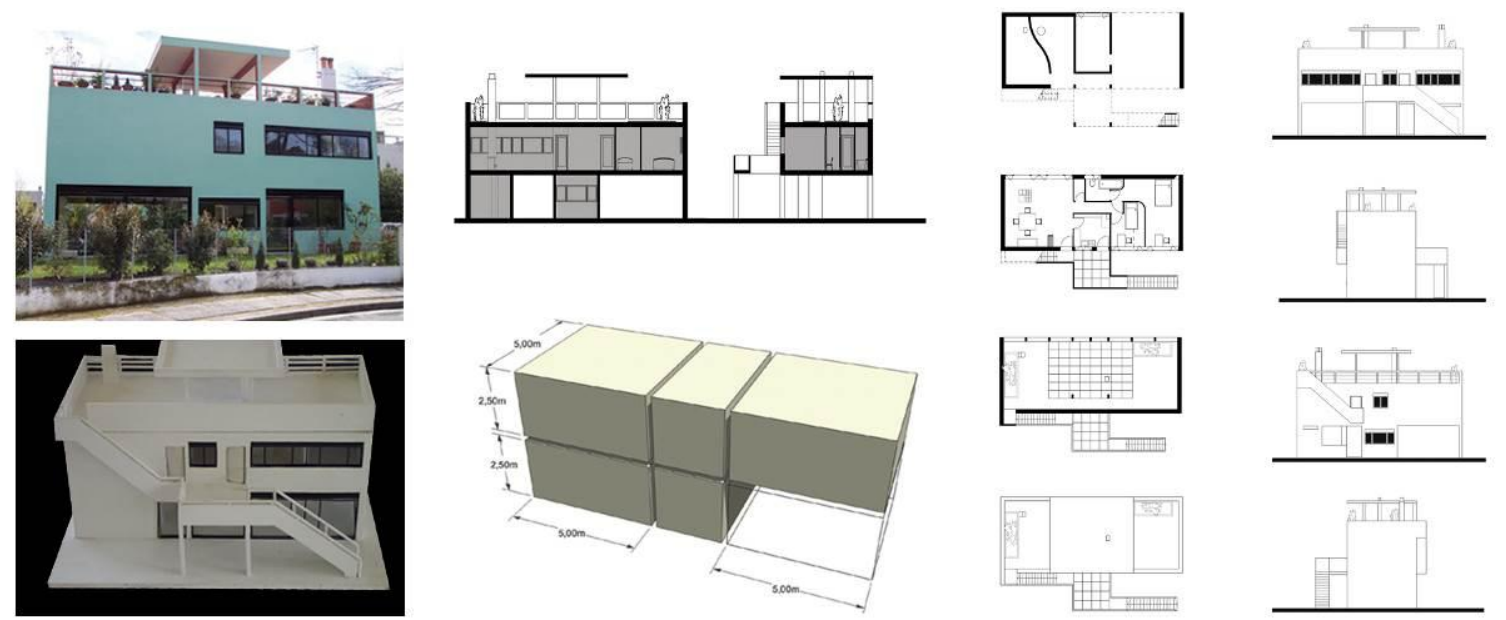

19. Cité Frugès, Les Maisons Vrinat travaux d'étudiants Studio Laurent Duport, ENSAM

Isolée :

L'escalier extérieur court le long de la façade et donne accès aux différentes pièces de vie (deux chambres, cuisine, séjour et salle de bains) situés au premier étage. Au rez-de-chaussée on trouve les pièces de services (garage, et chai). En tout $70 \mathrm{~m}^{2}$ de surface habitable. La façade est verte et blanche, les parois latérales extérieures à l'escalier, et celles intérieures à l'escalier ainsi que la terrasse sont terre de Sienne.

\section{Transformations}

La lecture attentive de l'ouvrage de Philippe Boudon «Pessac de Le Corbusier » dans son édition de 1985 nous offre de précieux éclairages sur la réception des Quartiers Modernes Frugès depuis leur construction. La présentation historique du projet, les réactions de la presse mais surtout l'analyse de la conception de Le Corbusier à Pessac et les interviews des habitants montrent que les modifications effectuées par les occupants «constituent un pas positif et non une conséquence négative de la conception originale de Le Corbusier. Pessac a non seulement permis aux occupants une latitude suffisante pour satisfaire leurs besoins, ce faisant, elle les a aussi aidés à réaliser quels étaient ces besoins $»^{18 .}$

\footnotetext{
${ }^{18}$ Huxtable Ada Louise, “Le Corbusier's housing project-flexible enough to endure” in New York Times, 15 Mars 1981
} 


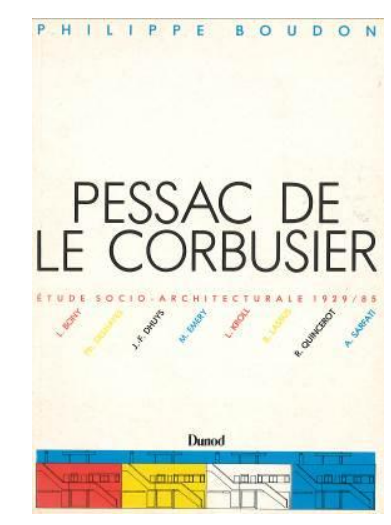

20. Couverture du livre de P.Boudon

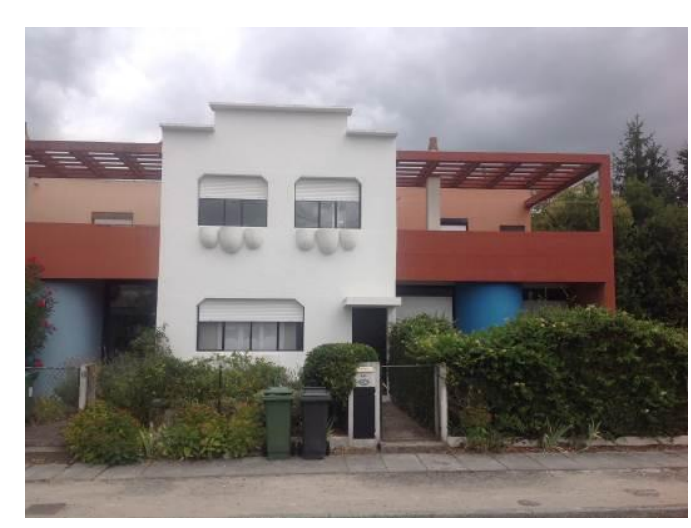

21. Une maison quinconce transformée

Dans la deuxième partie de l'ouvrage, les témoignages d'Alain Sarfati et de Laurent Bony sont particulièrement importants à l'aune du processus de patrimonialisation qui s'annonce à la fin des années 90. Ainsi pour Sarfati Pessac aura été une très grande leçon et «dans le cas de Pessac, toute intervention apparaissait comme une altération de l'œuvre, et pourtant l'histoire nous enseigne que les grandes auvres sont aussi celles qui ont été capable de subir des extensions des transformations. Aujourd'hui au cour du débat sur l'architecture urbaine, sur les morceaux de ville où la politique de quartier, Pessac reste un exemple à explorer. Une des notions clés de l'architecture moderne et celle d'espace une notion qui a alimenté la spécificité du travail des architectes. L'espace, difficile à saisir, à décrire, à qualifier, cet espace de l'architecture est toujours un espace intérieur qui oublie le grand espace qui en résulte, l'espace qu'elle constitue de fait, l'espace public. À Pessac, s'il y a un espace public ce sont les habitants qui l'ont fabriqué tant bien que mal et c'est l'occasion de s'interroger sur les différents couples d'opposition public / privé, intérieur / extérieur, mais aussi sur ce couple d'opposition étonnant architecture / nature. D'une certaine manière, Pessac constitue le lieu idéal pour produire une introduction à l'architecture urbaine et donc un autre regard sur la ville comme espace complexe et non plus simplement fonctionnel ${ }^{19}{ }^{1}$.
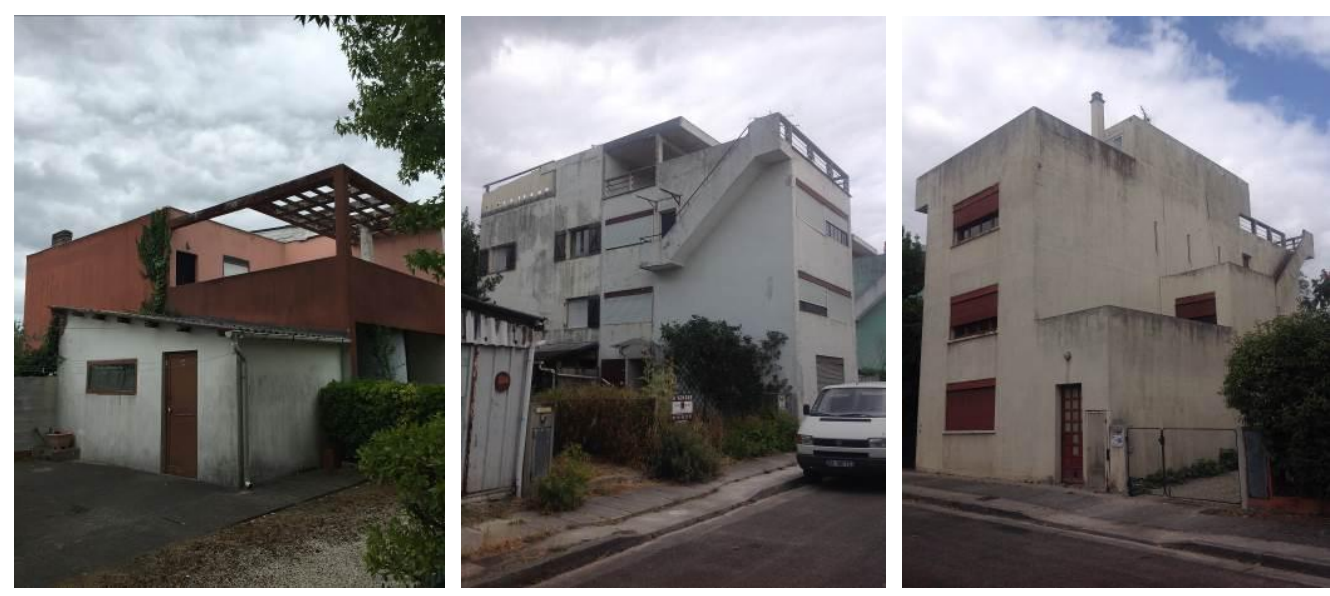

22 - 23 - 24. Quelques maisons transformées.

Pour Laurent Bony la leçon de Pessac est encore plus évidente : "Il faut voir en Pessac, ni un échec ni une réussite incontestable, encore moins incontestée mais simplement un groupe de maisons qui a fait l'objet d'un projet d'architecte, qui a été construit, qui est habité. En cela c'est à la fois ordinaire et exemplaire. Si l'on se rend à Pessac pour y voir ce qui est représenté dans les Euvres Complètes on sera déçu., Si l'on veut y voir un

\footnotetext{
${ }^{19}$ Boudon Philippe, Pessac de Le Corbusier, p.206
} 
quartier vivant construit par le Corbusier et par ses habitants, on constatera que la réalité concrète des maisons a permis, plus ou moins facilement, une appropriation progressive, des transformations liées à l'usage et à la signification de la maison; que les transformations ont le plus souvent utilisé une opportunité construite, une accroche physique, une amorce de marquage existant dans le projet de Le Corbusier. Ces transformations dépassant parfois le projet plutôt qu'elles ne le contredisent.(...) Avec Pessac émerge de toute évidence un conflit entre les recherches de Le Corbusier en 1925 et les préférences architecturales immédiates des habitants; mais il y a surtout des traces concrète de la rencontre entre le travail d'un architecte et la pratique des utilisateurs. L'observation des rajouts et transformation, l'appropriation générale de leur quartier par les habitants intervenant sur chacune de leur maison, nous renseignent sur la signification de la maison, l'ordre domestique, le statut des espaces extérieurs» ${ }^{20}$

\section{Patrimonialisation}

Inoccupées pendant plus de deux ans après leur livraison, aucune maison ne sera vendue car les raccordements aux réseaux de gaz, d'eau et d'électricité ne sont pas terminés. Les maisons achetées par les premiers propriétaires (seulement en 1929/1930 dans le cadre de la loi Loucheur ${ }^{21}$ ), restées vides, ont déjà subi des détériorations. La guerre va accentuer la dégradation de l'aspect d'origine des QMF avec le bombardement de la voie ferrée qui détruit deux maisons et brise la plupart des vitrages. Leur remplacement va entraîner, bien souvent, le changement des menuiseries et le rebouchages de certaines baies, le bois prenant généralement la place des profilés métalliques d'origine. Les réparations, souvent à l'économie des étanchéités des toitures terrasses avec de la tôle ondulée ou d'autres matériaux disparates ainsi que la recherche d'une optimisation/augmentation de surfaces dans ces maisons aux dimensions standardisées, transforment le quartier au fil des années.

Ainsi après une longue période au cours de laquelle les maisons se dégradent et sont l'objet de toutes sortes de transformation avec des démolitions, des extensions, mais surtout des adaptations aux besoins et aux goûts de leurs habitants, il faut attendre 5 mars en 1973 avec la restauration de la maison située au numéro trois de la rue des arcades pour appréhender là une première reconnaissance patrimoniale avec son classement au titre des Monuments Historiques en 1980.

Ce classement induit un rayon de protection de $500 \mathrm{~m}$ autour de ladite maison et donc un contrôle des Monuments Historiques sur toutes les interventions apparentes sur le bâti des maisons de l'ensemble des Quartiers Modernes Frugès.

Parallèlement la municipalité de Pessac amorce la sauvegarde du quartier en réalisant une série de travaux de l'espace public: voirie, réseaux, plantations sont rétablis de la manière la plus conforme possible projet d'origine, et en 1983 achète une maison (un demi «gratte-ciel » au 4, rue Le Corbusier) et la restaure en « Maison Le Corbusier » initiant ainsi un chantier expérimental de Septembre 1987 à mars 1988.

«En 1985, une étude évaluera le niveau de dégradation de la cité par rapport à son état d'origine et recommandera des solutions architecturales techniques à mettre en ceuvre pour la restauration. $»^{22}$

\footnotetext{
${ }^{20}$ Ibid p. 177

${ }^{21}$ Loi du 13 juillet 1928permettant à un particulier d'emprunter à taux réduit afin d'acheter un terrain et d'y faire construire un pavillon ou une maison

${ }^{22}$ Ferrand Marylène, Feugas Jean Pierre, Le Roy Bernard, Veyret Jean Louis, Le Corbusier : Les Quartiers Modernes Frugès p.113
} 
Ainsi: «l'un des problèmes cruciaux de la cité consiste en l'état actuel de son gros ouvre, et tout particulièrement des ouvrages béton : des murs de façade fissurés avec affleurement ou mise à nu des armatures en acier, des gardes corps, des linteaux, des appuis éclatés et boursouflés, des pergolas et des escaliers menaçant de rupture. De gros problèmes sont à noter aussi au niveau de l'étanchéité des terrasses sur la plupart des maisons, ainsi que des problèmes d'imperméabilité au niveau des menuiseries métalliques.

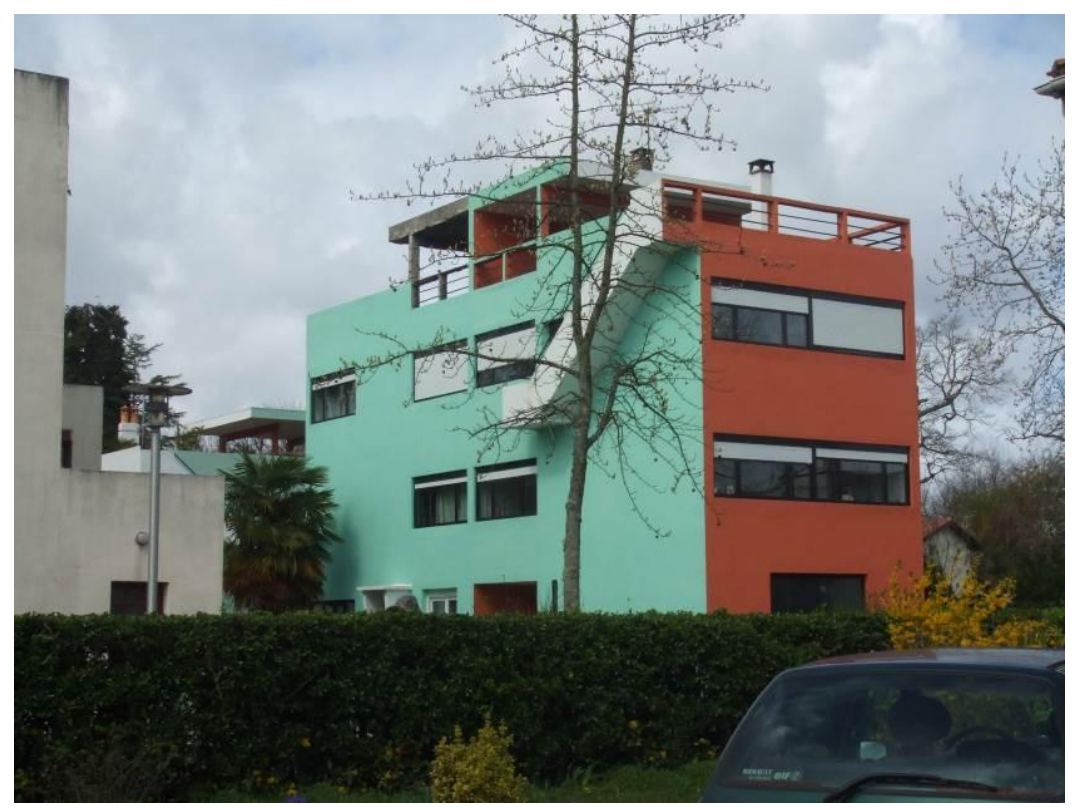

25. Cité Frugès, La maison Gratte-ciel au 4 rue Le Corbusier (Maison Municipale)

Nous pensions que face à cette dégradation et aux transformations tendant à faire disparaître l'œuvre de Le Corbusier, il s'agissait essentiellement de sauver les quartiers modernes Frugès, sans en chasser les habitants. Comment sauvegarder cette ensemble urbain, homogène exemplaire, tout en y associant ses habitants, en les motivant et en les sensibilisant à des actions de restauration, de réhabilitation et de rénovation raisonnées?

La Cité Frugès à Pessac est un monument historique moderne, habitée par une population qui désire y vivre, non pas comme dans un musée, mais comme dans un lieu vivant, en le pratiquant, l'entretenant et l'améliorant.

Il fallait donc élaborer une politique de sauvegarde définie par des recommandations détaillées et des prescriptions particulières. L'alternative n'était pas entre tout conserver ou tout détruire, c'est-à-dire entre la reconstitution fidèle à l'état d'origine ou le renoncement à toute intervention. Il existait une marge de manouvre dans laquelle pouvait s'inscrire des interventions architecturales pertinentes basées sur une armature théorique simple permettant la sauvegarde de cet ensemble historique, tout en conservant le caractère de cité populaire.(...)

Les maisons de Pessac étant, malgré toutes les tentatives d'industrialisation, des constructions composites associant substances et matériaux de longévités variables, le renouvellement cyclique de certaines parties est dans la nature même des choses. Dans tous les cas possibles, on cherchera alors la conservation de la substance ancienne, l'état actuel des techniques permettant de trouver des solutions de réparation et de rajeunissement satisfaisantes. Dans les cas de remplacement intégral des parties manquantes, de matériaux irrécupérables ou d'éléments inefficients, les éléments destinés à les remplacer devront s'intégrer étroitement à la composition de l'ensemble et respecter le plus fidèlement possible la nature et le dessin formel prédominant. Cette clause est en effet parfaitement tenable intellectuellement, techniquement et économiquement, compte tenu, d'une part, de la 
formation très complète et maîtrisée que l'on a sur l'état d'origine, d'autre part, des capacités technologiques de production et de reproduction que l'on possède aujourd'hui et qui concerne des appareils ou ouvrages connus et relativement sophistiqués. Les solutions architecturales projetées, pour améliorer ou adapter des situations techniques défaillantes constituant la cause des dégradations, devront, dans leur composition, rester aussi discrète que possible et toujours garantir la clause de réversibilité de l'intervention. Enfin ces interventions de conservation ne doivent pas faire oublier qu'elles imposent d'abord la permanence de l'entretien de l'œuvre ». ${ }^{23}$

Par la suite des propriétaires individuels aidés par un système de subventions ainsi que l'OPAC Aquitanis, avec l'acquisition de quatre maisons en 1993 \& 1994 prolongent l'intervention municipale et permettent de préfigurer ce que peut redevenir l'ensemble des quartiers modernes.

Ces initiatives sont confortées par l'élaboration de la Zone de Protection du Patrimoine Architectural, Urbain et Paysager (ZPPAUP) des Quartiers modernes Frugès, créée le 27 octobre 1998 qui énonce les règles constructives à respecter et des conseils pour protéger et mettre en valeur les logements conçus et réalisés par Le Corbusier et Pierre Jeanneret de 1924 à 1927

"Face à la dégradation pathologique et aux transformations tendant à mutiler l'œuvre de Le Corbusier et Pierre Jeanneret, comment sauver les Quartiers modernes Frugès sans en chasser une population qui désire y vivre, non pas comme dans un musée, mais comme dans un lieu vivant en l'entretenant et en l'améliorant". La ZPPAUP des Quartiers modernes Frugès créée permet de répondre à cette problématique. ${ }^{24}$

Ainsi le caractère exemplaire et didactique des Quartiers Modernes Frugès n'est plus à démontrer aussi bien dans son aspect théorique, que sur le caractère de témoignage d'une époque qu'il représente. La valeur ajoutée réside dans le processus de patrimonialisation dont la Cité Frugès a fait l'objet permettant à la fois la reconnaissance d'un ensemble architectural et urbain homogène d'une époque et l'élaboration d'une doctrine contemporaine de restauration qui s'appuie sur trois domaines d'études : le champ architectural, le champ technologique et le champ sociologique.

\section{Aujourd'hui}

Dans le cadre de l'opération de restauration des toitures et façades des maisons des Quartiers Modernes Frugès, la Ville de Pessac accompagne les propriétaires dans leurs projets de réhabilitation par le biais d'un dispositif d'aide administrative, technique et financière et a mis en place en 2011 un dispositif de guichet unique.Il s'agit d'une part de faciliter l'accès aux aides financières pour les propriétaires désireux de réaliser des travaux, mais aussi d'accélérer et d'harmoniser les réhabilitations.

Ce fonds mutualise les aides de la Ville de Pessac et de la Région Aquitaine à parts égales (10 $500 €$ pour chaque partenaire), la DRAC Aquitaine, le Conseil Général de la Gironde et la Fondation du Patrimoine participant également à cette opération selon leurs propres modalités.

D'autre part, la Région et la Ville de Pessac, ont engagé une réflexion commune sur la valorisation des travaux réalisés sur les maisons et sur la cité Frugès, par l'intermédiaire d'une publication dans la collection «Visages du Patrimoine en Aquitaine » dont le partenariat est formalisé jusqu'au 31 décembre 2015.

\footnotetext{
${ }^{23}$ Rencontres de la Fondation Le Corbusier, « La conservation de l'œuvre construite de Le Corbusier », collectif, Fondation Le Corbusier, Paris, 1990, p.74-87

${ }^{24}$ Présentation de la ZPPAUP par la DRAC Aquitaine : http://aquitaine.culture.gouv.fr/dossiers-thematiques/architectureurbanisme/zppaup
} 

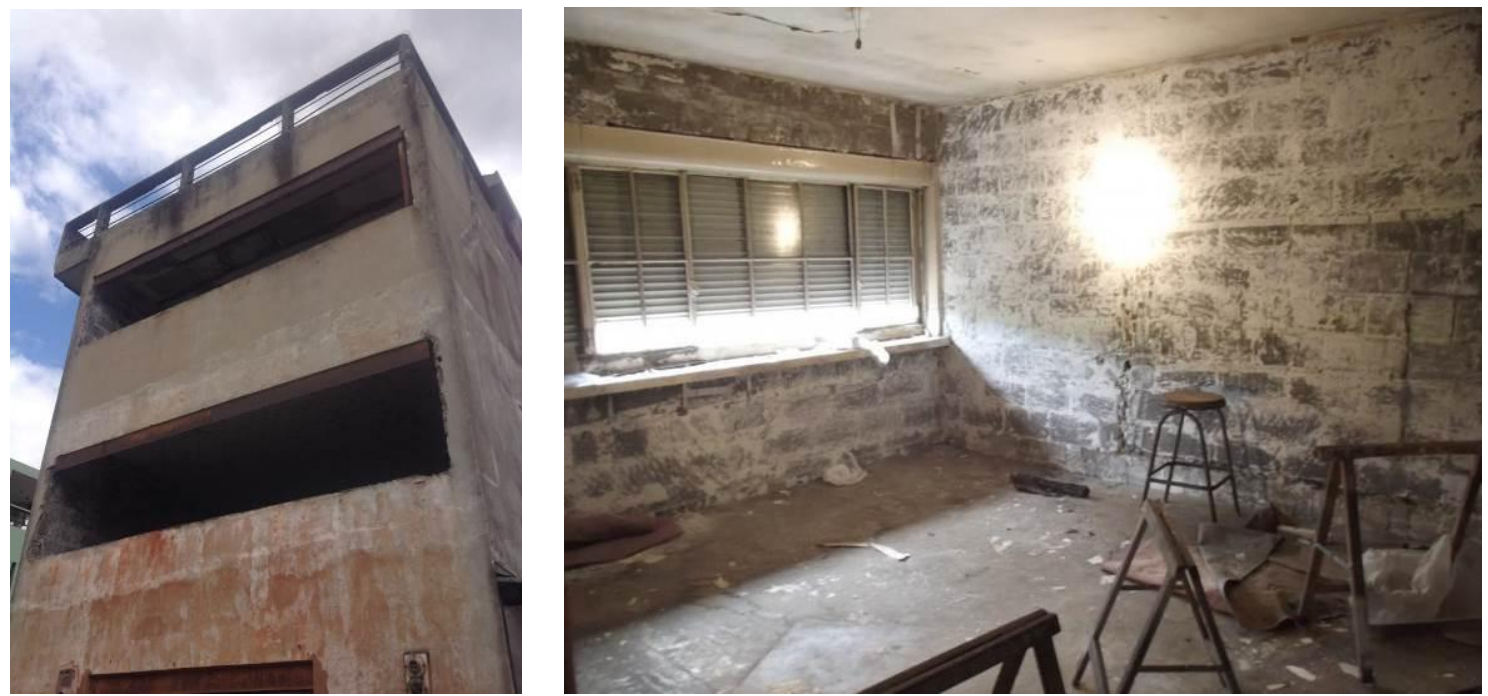

26. Cité Frugès, La maison Gratte-ciel au 12 rue Le Corbusier

27. Cité Frugès, La maison Gratte-ciel au 12 rue Le Corbusier

Du point de vue du marché immobilier, dans son article de Médiapart David Gauthier écrit : «La poussière s'élève à chaque pas. Les deux agents immobiliers viennent de pénétrer dans le rez-de-chaussée de cette maison de type «gratte-ciel ». Elle est en vente, et ils me la font visiter. Ils montent à pas lents le large escalier de béton qui zèbre l'habitation sur ses trois étages. La pièce à vivre est sombre. Impossible de se figurer à quoi ressemblait la vie ici : il ne reste rien des aménagements d'origine. Un des agents parvient à ouvrir de quelques centimètres les volets roulants, laissant filtrer un trait de lumière. Le petit salon apparaît plus nettement. Son état de délabrement est avancé. Le papier peint n'a pas survécu aux affres du temps. Il n'en reste que des lambeaux. "Il faut compter environ 300000 euros pour les travaux, préviennent-ils. En plus des 600000 euros pour acquérir les deux maisons ${ }^{25}{ }$.

En 2015 la Cité Frugès fait l'objet de nombreuses visites par les étudiants, architectes, visiteurs et touristes ou simples curieux, et ce, tout au long de l'année.

La maison " gratte-ciel » acquise en 1983 par la Ville de Pessac a bénéficié d'un chantier de rénovation expérimental exemplaire permettant une cohérence globale du point de vue esthétique et de l'aménagement de l'espace. Classée au titre des Monuments historiques, la Maison Frugès-Le Corbusier accueille «un public toujours plus nombreux, composé tant du grand public que des regards plus avertis répondant, d'une certaine manière, à une tendance de la société, plus intéressée par l'habitat et son évolution.Véritable support d'éducation du regard à l'architecture moderne, elle est aussi source d'inspiration pour de nombreux artistes et se convertit en lieu d'exposition 4 fois par an, déclinant les thématiques en lien avec l'aspect laboratoire de la Cité : l'architecture moderne, l'urbanisme, l'art contemporain, le design... Elle est aussi un lieu ressource pour les chercheurs, étudiants ou les propriétaires de la cité dans le cadre de la restauration de leur maison ${ }^{26}$.

\footnotetext{
${ }^{25}$ Gauthier David, Cité Frugès : « La difficile empreinte de Le Corbusier à Pessac » article in Médiapart, 09 Février 2015

${ }^{26}$ Présentation de la Cité Frugès par la Ville de Pessac (www.pessac.fr)
} 

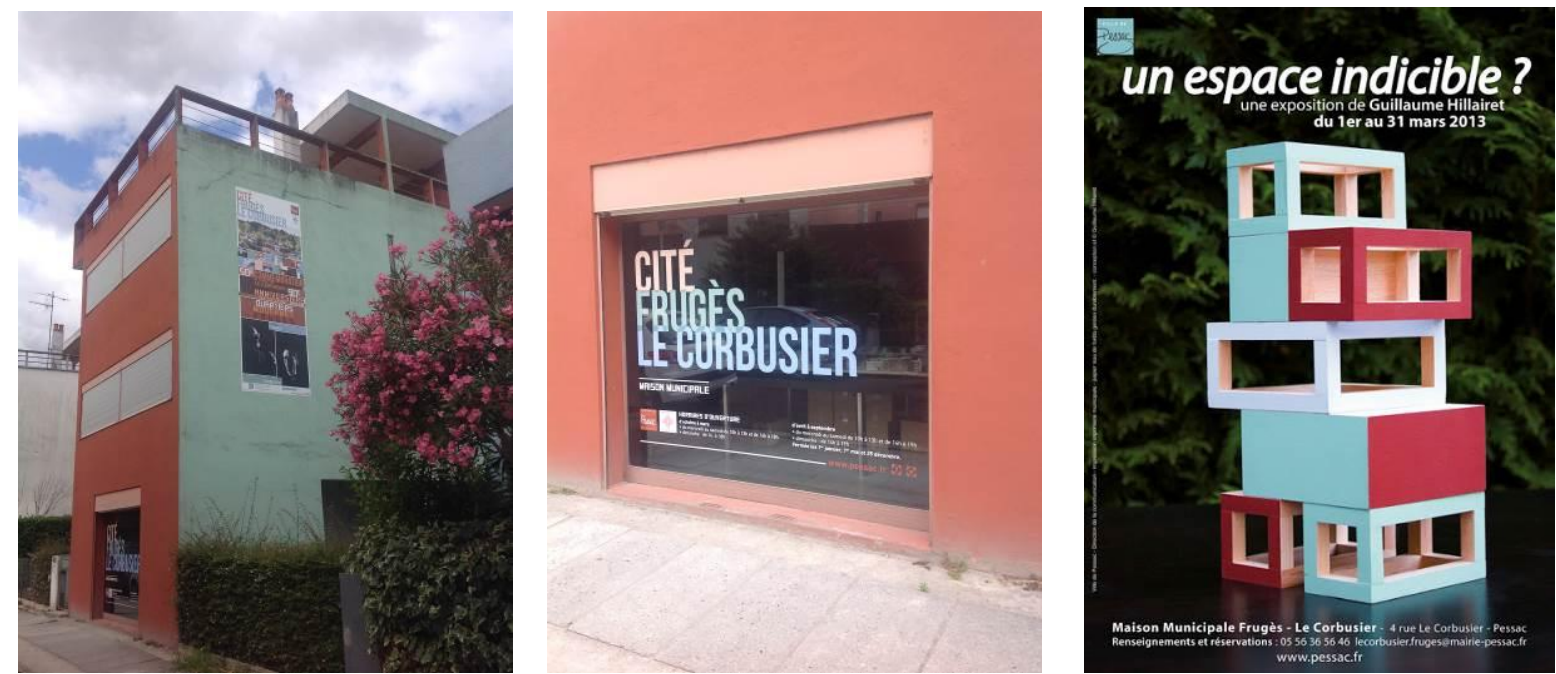

28. - 29. Cité Frugès, La maison Gratte-ciel au 4 rue Le Corbusier (Maison Municipale)

30. Affiche de l'exposition de Guillaume Hillairet à La maison Gratte-ciel du 4 rue Le Corbusier

Pour sa part Cyril Zozor médiateur du patrimoine y travaille depuis 2009 et passe ses journées au contact des visiteurs et des habitants qu'il aide pour valoriser leurs maisons conçues par l'architecte.

"Vous êtes passionné!», entend-il souvent au moment de dire au revoir à ses hôtes. À chaque visite commentée de la cité Frugès-Le Corbusier, c'est un peu la même histoire. Le public boit les paroles du guide, contant mille et une anecdotes sur les maisons bleu et terre de Sienne gratte-ciel, quinconce, arcade ou zigzag, leurs usages et leurs habitants au fil du temps. Son travail consiste à accueillir groupes scolaires et visiteurs, avertis ou non, mais aussi à épauler les habitants de la cité pour protéger, conserver et valoriser leur maison. "C'est un endroit vivant, pas un musée. C'est toute la richesse et la complexité du site. Qu'est-ce qu'on peut faire de manière intelligente pour conserver et continuer à y vivre? Le Corbusier avait du génie, il était en avance sur son temps. Il ne l'est plus. Nos modes de vie ont changé, explique-t-il. Ces habitations étaient conçues pour avoir 300 objets. Nous en avons 3000 aujourd'hui dans nos foyers. Regardez les fenêtres, conçues telles des pellicules cinématographiques. Elles sont magnifiques mais pas pratiques du tout. Comment voulez-vous les laver ? ${ }^{27}$

\section{Transmission}

Parmi les nombreux collaborateurs de Le Corbusier, seule une demi-douzaine d'architectes ont été professeurs avec une forte influence sur des générations d'architectes et dans certaines écoles d'architecture. Il s'agit de José Luis Sert (1902-1983) et Jerzy Soltan (1913-2005), de Georges Candilis (1913-1995), de Guillermo Jullian de la Fuente (1931-2008) et de José Oubrerie (né en 1932). Cette implication se traduit selon trois périodes distinctes.

Sert et Soltan ont été les «piliers » de la mise en place de la Graduate School of Design à Harvard. Sert est nommé Dean dès 1953 jusqu'en 1969 et imprimera une forte empreinte sur plusieurs générations d'architectes. Sert sera également associé à Le Corbusier pour la construction du Carpenter Center for Visual Arts, seule réalisation construite par Le Corbusier aux Etats Unis. Soltan, lui est nommé Robinson Professeur of Architecture and Urban Design dès 1959 jusqu'en 1979.

\footnotetext{
${ }^{27}$ Bosdecher Laurie « Le prof devenu médiateur » article in Sud-Ouest, 13 Août 2012
} 
Georges Candilis lui a eu une forte influence sur l'enseignement de l'école des Beaux-Arts en France dès 1965 et sur son évolution avec la création des Unités Pédagogiques d'Architecture, notamment l'UPA n ${ }^{\circ} 6$ après Mai 1968. Il va enseigner lui aussi jusqu'à la fin des années 70 .

Jullian et Oubrerie, quant à eux ont plusieurs points communs. «Le premier et qui sont parmi les derniers collaborateurs de l'atelier du 35 rues de Sèvres, donc parmi ceux qui ont quotidiennement côtoyé le Corbusier dans les dernières années de sa vie : dans cette mesure ils sont déjà complices. Le second est qu'après cet épisode qui les a bien sûr marqués, ils ont traversé une période de plus de dix années pendant laquelle ils ont eu diverses activités qui ne les ont pas immédiatement menés à la réalisation d'édifices importants. Ils ont d'abord tenté de faire exister ce dont ils héritaient : des travaux commencés avec le Corbusier et laissés inachevés. Ainsi Guillaume Jullian poursuivit le projet pour le nouvel hôpital de Venise, et José Oubrerie le projet pour l'église de Firminy ${ }^{28}$ qu'il réalisera et achèvera finalement en 2006.

Par la suite dans leurs premiers projets construits, José Oubrerie à Damas et Guillaume Jullian à Rabat, «affichent clairement leurs préoccupations : construire l'espace architectural par le volume et la lumière, et offrir les moyens de la compréhension de cette construction, sans que jamais ceci ne soit donné de façon ostensible : que l'émotion architecturale puisse participer d'une découverte, D'une compréhension, d'une intelligence de l'œuvre $»^{29}$.

Après une période d'enseignement en France, Jullian et Oubrerie décident d'émigrer aux Etats Unis et y enseignent à partir du milieu des années 80 en liant des amitiés avec des personnalités comme John Hejduk, Robert Slustky ou Kenneth Frampton. Jullian est professeur invité dans les universités de MIT, Harvard, Cornell, University of Pennsylvania jusqu'en 2003, Oubrerie est lui aussi invité dans les universités de Columbia University, Cooper Union, de Lexington (où il sera Dean et Professeur de 1987 à 1991) puis à Columbus (Head of Architecture and Professeur de 1991 à 2013).

\section{D'autres personnalités}

Tadao Ando est parti de la passion corbuséenne d'un de ses étudiants pour entreprendre avec ses élèves de l'Université de Tokyo la modélisation, à la même échelle (1:200), de toutes les maisons conçues et réalisées (ou non) par Le Corbusier (il en dénombre 106 !). Ce travail a fait l'objet d'une publication ${ }^{30}$ et d'un don à la Fondation Le Corbusier et les maquettes ont été exposées dans différents lieux (Galerie Patrick Seguin, Paris en 2006, Placé le radieux en $2011 \ldots$ )

\footnotetext{
${ }^{28}$ Lucan Jacques, "Deux anciens de chez Corbu" article in AMC n 17 , Octobre 1987 p.52-71

${ }^{29}$ Ibid

${ }^{30}$ Ando Tadao Laboratory « Le Corbusier HOUSES », Toto, Tokyo, 2001
} 

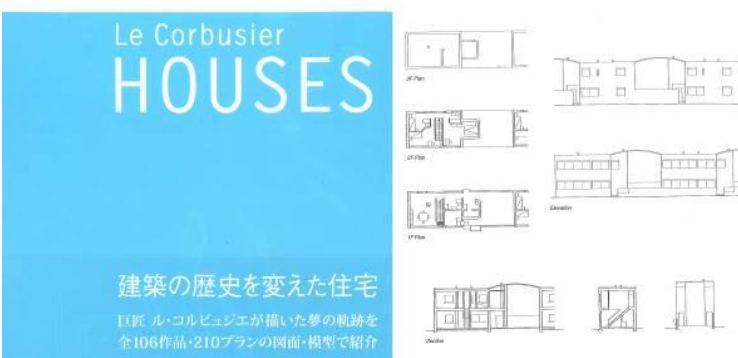

31. Couverture et page 138 du livre de Tadao Ando

Henri Ciriani a enseigné en France de 1969 à 2002 d'abord seul, puis à partir de 1979 au sein d'un collectif, le groupe $\mathrm{UNO}^{31 .}$ Pour lui la pédagogie c'est «apprendre aux étudiants à courir un cent mètres». Et cet entrainement se fera dans l'univers formel de "l'espace moderne », entendu comme prolongement de l'œuvre de Le Corbusier ${ }^{32}$. Ainsi sont développés des exercices comme : le logis, une manipulation de la géométrie d'un carré de 10X10 mètres soumis à un programme et "l'espace 30X30 ou l'apprentissage du plan libre: une bibliothèque dont la structure est constituée d'une trame de poteaux de 5 à $6 \mathrm{~m}$ de portée. Il s'agit d'une bibliothèque sur un espace carré de 30X30 mètres où la compacité de la figure oblige, par incapacité de la périphérie à éclairer le centre, à trouver une spatialité particulière pour la lumière de la partie centrale ${ }^{33}$ »
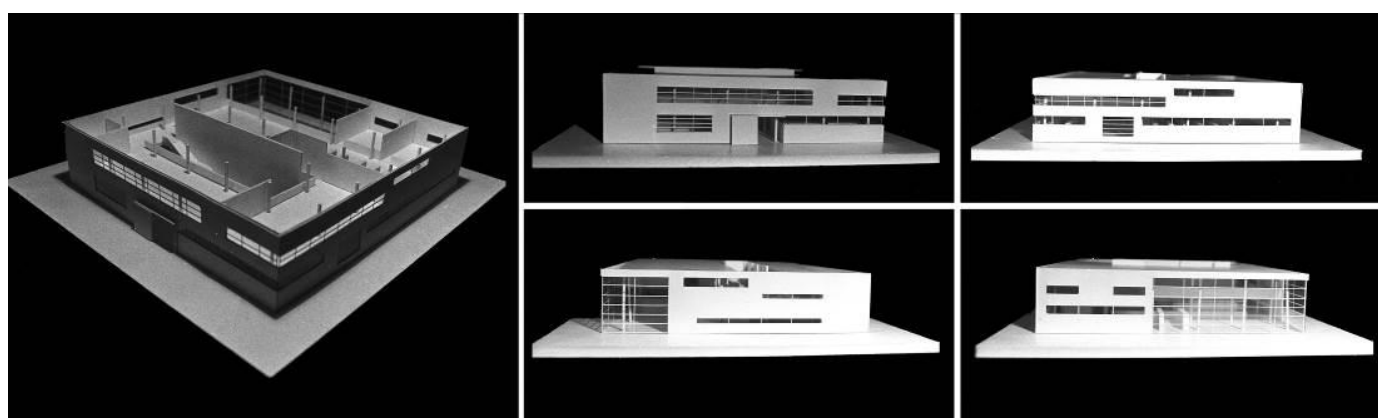

32. L'espace $30 X 30$ une bibliothèque, Laurent Duport ENSAPB 1988

Peter Eisenman travaille depuis de nombreuses années avec ses étudiants de l'université de Yale sur Le Corbusier. En 2014 cela s'est traduit à travers un séminaire qui proposait une discussion très spécifique sur le travail de Le Corbusier sur la base de deux de ses écrits fondamentaux : les cinq points de l'architecture et les quatre compositions. Ces textes sont lus à travers l'analyse et le dessin d'une série de bâtiments spécifiques et leurs évolutions de 1914 à 1965 et sont complétés par des lectures plus générales ainsi que d'autres points de vue de théoriciens comme Stanislaus von Moos, Anthony Vidler, Kurt Forster et Pier Vittorio Aureli.

\footnotetext{
${ }^{31}$ Arnold Françoise et Cling Daniel, « Transmettre en architecture. De l'héritage de Le Corbusier à l'enseignement d'Henri Ciriani », , Le Moniteur, Paris, 2002 p.194-201

${ }^{32}$ L'Architecture d'Aujourd'hui Henri Ciriani Septembre 1992 n ${ }^{\circ} 282$

${ }^{33}$ L'Architecture d'Aujourd'hui, Le Corbusier, Février 1987, p.68-72
} 


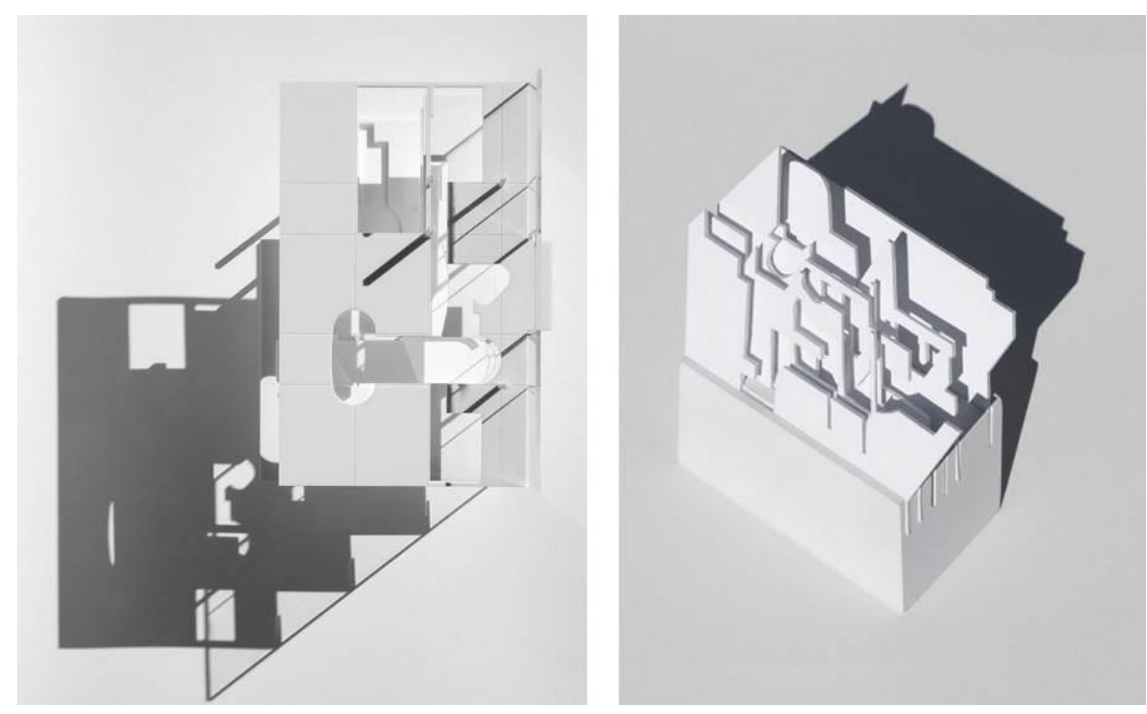

33. Travaux d'étudiants de Peter Eisenman à YSOA, Diagrammatic Modernism

Franz Graf avec ses étudiants de troisième année du Bachelor à l'EPFL, organise chaque année un atelier de techniques et sauvegarde de l'architecture moderne. «Le projet de sauvegarde et le projet dans l'existant font partie d'une discipline culturelle constituée qui élargit le projet d'architecture contemporain avec des questionnements théoriques et pratiques sur l'objet architectural à toutes ses échelles ainsi que le regard rapproché sur la matérialité du bâti qui donnent naissance au projet ${ }^{34}$ Pour l'année 2014 l'atelier a porté sur les Quartiers Modernes Frugès de Le Corbusier à Pessac, sauvegarde et devenir d'une cité d'habitation
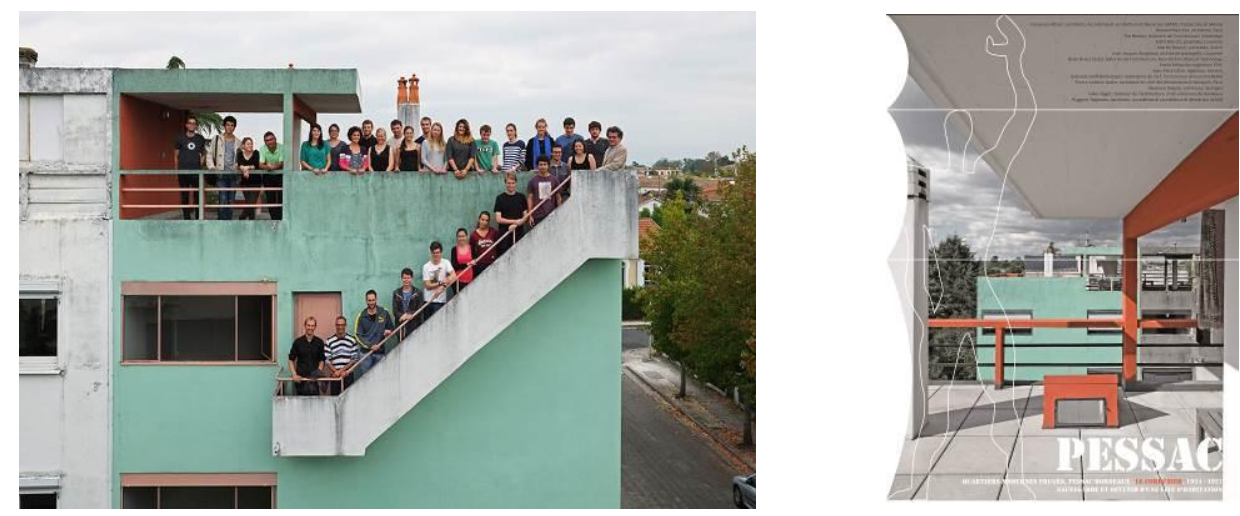

34. Affiche et l'atelier de Franz Graf à l'EPFL

\section{Si je devais enseigner l'architecture?}

Lors d'une conférence donnée à Buenos Aires en 1929, devant un auditoire de professionnels composé d'architectes, d'ingénieurs et d'étudiants en architecture, Le Corbusier répondit :

«Si je devais enseigner l'architecture? Je commencerais par interdire les "ordres», par faire cesser cette maladie des "ordres», le scandale des "ordres», cette inimaginable défaillance de l'esprit. J'exigerais: respect à l'architecture. Je m'efforcerais à inculquer à mes élèves le sens aigu du contrôle, du libre arbitre, du «comment et du « pourquoi ». Je leur enseignerais le mépris des formules. Je leur dirais : tout est rapports.(...)

\footnotetext{
${ }^{34}$ Présentation de l'atelier Graf à l'EPFL : http://tsam.epfl.ch/
} 
Avant de dessiner il faut toujours savoir « de quoi il s'agit », «à quoi ça sert », "pour quoi c'est faire ». Enfin il faut apprendre à regarder $»^{35}$

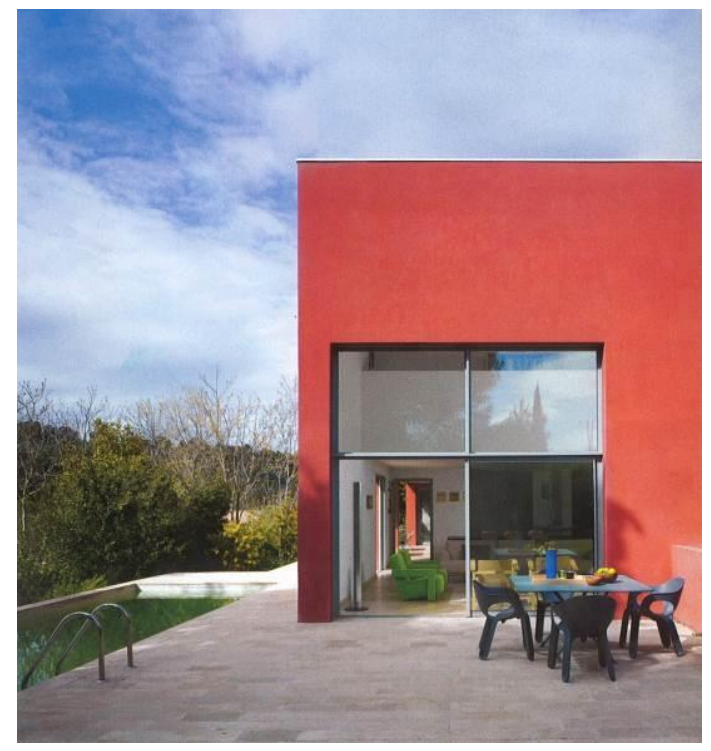

35. Tribute to Le Corbusier, Villa CLMT, Nîmes, Laurent Duport architecte.

\section{Acknowledgements}

Ines Zalduendo, Special Collections, Frances Loeb Library / Harvard University GSD

Shelley Hayreh, Avery Architectural and Fine arts Library, Columbia University

Students of Studio Laurent Duport "Urban Grafts", Ecole Nationale Supérieure d'Architecture de Montpellier, 2006-2015

\section{Sources of Images :}

Image $1:$ tirée de «Vers une architecture » p.212

Image 2 : tirée de «Vers une architecture » p.213

Image 3 : Quartiers Modernes Frugès tirée de l'Architecture Vivante, 1927

Image 4 : tirée du livre de Philippe Boudon, Pessac de Le Corbusier, p.38

Image 5 : tirée de Le Corbusier Euvres Complètes 19101929 p.69

Image 6 : tirée de Le Corbusier Euvres Complètes 19101929 p.77

Image 7 : Le Corbusier et Pierre Jeanneret, Quartiers Modernes Frugès, Pessac, étude de polychromie, 1924. Gouache sur calque, 78,2 X $110 \mathrm{~cm}$. Fondation Le Corbusier

Image 8 : Idem, Etude de polychromie, 1924. Gouache sur calque, 41,8X114,2 cm. Fondation Le Corbusier Image 9 : Quartiers Modernes Frugès Etude des couleurs tirée de l'Architecture Vivante, 1927

Image 10 : Polychromie architecturale Eventail des 63 « couleurs Salubra » de Le Corbusier Image 11 : Couleurs de la Cité Frugès issues de Eventail des 63 «couleurs Salubra » de Le Corbusier Image 12 : Maisons Jaoul, Le Corbusier, Maison A, salon, photographie Jean Christophe Pratt

\footnotetext{
${ }^{35}$ Guiton Jacques Le Corbusier, Textes choisis Architecture et urbanisme, Le Moniteur, Paris 1982
} 
Image 13 : Maisons Jaoul, Le Corbusier, Maison A, entrée, photographie Jean Christophe Pratt Image 14 : Extrait du dépliant accompagnant l'exposition «L'aventure Le Corbusier », Paris 1987 Image 15 : Cité Frugès, Les Maisons Gratte Ciel travaux d'étudiants Studio Laurent Duport, ENSAM Image 16 : Cité Frugès, Les Maisons Arcades travaux d'étudiants Studio Laurent Duport, ENSAM Image 17 : Cité Frugès, Les Maisons Quinconces travaux d'étudiants Studio Laurent Duport, ENSAM Image 18 : Cité Frugès, Les Maisons Zig Zag travaux d'étudiants Studio Laurent Duport, ENSAM Image 19: Cité Frugès, Les Maisons Vrinat travaux d'étudiants Studio Laurent Duport, ENSAM Image 20 : couverture du livre de P.Boudon

Image 21 : une maison quinconce transformée, photographie Laurent Duport Image 22 : une maison quinconce transformée, photographie Laurent Duport Image 23 : une maison Gratte Ciel transformée, photographie Laurent Duport Image 24 : une maison Gratte Ciel transformée, photographie Laurent Duport Image 25 : La maison Gratte-ciel au 4 rue Le Corbusier (Maison Municipale) photographie Laurent Duport Image 26 : Cité Frugès, La maison Gratte-ciel au 12 rue Le Corbusier photographie Laurent Duport Image 27 : Cité Frugès, La maison Gratte-ciel au 12 rue Le Corbusier photographie David Gauthier Image 28 : La maison Gratte-ciel au 4 rue Le Corbusier (Maison Municipale) photographie Laurent Duport Image 29: La maison Gratte-ciel au 4 rue Le Corbusier (Maison Municipale) photographie Laurent Duport Image 30 : Affiche de l'exposition de Guillaume Hillairet à La maison Municipale, Ville de Pessac Image 31 : Couverture et page 138 du livre Le Corbusier HOUSES de Tadao Ando Image 32 : L'espace 30X30, Photographies maquette Fond Laurent Duport Image 33 : Travaux d'étudiants de Peter Eisenman à YSOA, Diagrammatic Modernism Image 34 : Affiche et l'atelier de Franz Graf à l'EPFL

Image 35 : Tirée de ARQUITECTURA Y DESIÑO n 91 Casas muy especiales, Manifesto Colorista p.151, photographie Eugeni Pons.

\section{Bibliography / references}

Ando Tadao Laboratory: Le Corbusier HOUSES, Tokyo: Toto, 2001

Arnold Françoise et Cling Daniel: Transmettre en architecture. De l'héritage de Le Corbusier à l'enseignement d'Henri Ciriani , Paris: Le Moniteur, 2002

Benton Tim, Pessac and Lège revisited : standards, dimensions and failures in Massilia, 2004 p.68

Bois Yve-Alain and others: De Stijl et l'architecture en France, Bruxelles: Mardaga, 1985

Bosdecher Laurie Le prof devenu médiateur Sud-Ouest, 13 Août 2012

Boudon Philippe: Pessac de Le Corbusier, première édition, Paris: Bordas, 1969, édition augmentée, Paris: Dunod, 1985

De Foville Alfred: Enquête sur les conditions de l'Habitation en France, Les Maisons Types, Paris 1894 
Duboy Philippe: Le Corbusier, Croquis de voyages et études, textes choisis, Paris: Louis Vuitton, 2009

Ferrand Marylène, Feugas Jean Pierre, Le Roy Bernard, Veyret Jean Louis: Le Corbusier : Les Quartiers Modernes Frugès, Paris: Fondation Le Corbusier, , Bâle, Boston, Berlin: Birkhäuser, 1998

Guiton Jacques: Le Corbusier, Textes choisis Architecture et urbanisme, Paris: Le Moniteur, 1982

Huxtable Ada Louise, Le Corbusier's housing project-flexible enough to endure New York Times, 15 Mars 1981

L’Architecture d'Aujourd'hui Henri Ciriani Septembre 1992 n²82

L’Architecture d'Aujourd'hui, Le Corbusier, Février 1987, p.68-72

Le Corbusier \& Pierre Jeanneret:Euvres Complètes 1910-1929 Zurich: Artemis, 1964

Le Corbusier: Vers une architecture, Éditions Crès, Paris: Collection de "L'Esprit Nouveau", 1923

Lucan Jacques, Deux anciens de chez Corbu AMC n ${ }^{\circ}$, Octobre 1987

Padovan Richard, towards universality, le corbusier mies +destijl, London \& New York: Routledge, 2002

Palazzolo Carlo, Vio Riccardo, In the footsteps of LE CORBUSIER, New York: Rizzoli, 1991

Ragot Gilles:Dion Mathilde: Le Corbusier en France, Paris: Le Moniteur, 1987

Sarkis Hashim: CASE: Le Corbusier's Venice Hospital and the Mat Building Revival, Munich-London-New York: Prestel Verlag, 2001

\section{Documents :}

Special Collections, Frances Loeb Library / Harvard University GSD:

Sekler Eduard \& Taylor Brian Brace Le Corbusier at Pessac, The Search for Systems and Standards in the Design of Low Cost Housing, Catalogue de l'exposition éponyme 12 0ctobre 30 Novembre 1972, Carpenter Center for the Visual Arts, Harvard University, en collaboration avec la Fondation Le Corbusier 